\title{
EFFECTS OF TREATMENT, AWARENESS AND CONDOM USE IN A COINFECTION MODEL FOR HIV AND HCV IN MSM
}

\author{
CARLA M. A. PINTO, ANA CARVALHO
}

\begin{abstract}
We develop a new a coinfection model for hepatitis C virus (HCV) and the human immunodeficiency virus (HIV). We consider treatment for both diseases, screening, unawareness and awareness of HIV infection, and the use of condoms. We study the local stability of the disease-free equilibria for the full model and for the two submod-els (HCV only and HIV only submodels). We sketch bifurcation diagrams for different parameters, such as the probabilities that a contact will result in a HIV or an HCV infection. We present numerical simulations of the full model where the HIV, HCV and double endemic equilibria can be observed. We also show numerically the qualitative changes of the dynamical behavior of the full model for variation of relevant param-eters. We extrapolate the results from the model for actual measures that could be implemented in order to reduce the number of infected individuals.
\end{abstract}

Keywords: Mathematical Models; HIV/AIDS; HCV; MSM; Screening Coinfection.

\section{Introduction}

Human immunodeficiency virus (HIV) affects 34 to 46 million people throughout the world. Of these, about 4 to 5 million people are coinfected with hepatitis $\mathrm{C}$ virus (HCV). ${ }^{1}$ In Portugal, it is estimated that about $25 \%$ to $40 \%$ of the 41,086 people infected with HIV are coinfected with $\mathrm{HCV} .{ }^{2}$ Portugal is the third country, of the Western Europe, with higher prevalence and more new diagnosed cases of HIV/AIDS, per year. 
The current international consensus, to control the HIV epidemic, focuses on the need for clear leadership on policies and programs for prevention, early diagnosis, treatment that respects human rights and quality of health care, effective and accessible to everyone. Mathematical models have been used extensively in the literature to model epidemiological and other biological phenomena. ${ }^{3,4}$ In what concerns HIV/AIDS, new models incorporate a more detailed understanding of the mechanisms associated to HIV infection, such as dissemination, and distribution and impact of interventions on population. ${ }^{5,6}$ Consequently, the impact of health policies, such as deficient access to health care, delayed treatment or the use of screening for asymptomatic cases can be evaluated with these models. ${ }^{7}$ A model of HIV/AIDS with screening was introduced in Ref. 8 and recently extended in Ref. 9. Usually, the models of the dynamics of HIV/AIDS, incorporate progression, where an infected individual goes through various stages of infectiousness before developing clinical AIDS. In Ref. 8, a model in which infection leads to asymptomatic HIV infectives, who are later screened and then develop AIDS, without interventions, is considered. A similar approach was previously studied in Ref. 5 with differential infectivity and staged progression models. In Ref. 9, the model in Ref. 8, was modified to include highly active antiretroviral therapy (HAART) treatment.

Typically, the coinfection of HIV and other diseases is associated with more serious risks and severe consequences for patients. In the coinfection with HCV, $\mathrm{HIV}$ accelerates the progression of $\mathrm{HCV}$, moreover, the risk of severe liver disease is higher if the CD4 count falls below 200 cells $/ \mathrm{mm}^{3} .^{2}$ In addition, there is a higher risk of cirrhosis, end-stage liver disease, hepatocellular carcinoma and liver-related death. ${ }^{10}$ In Portugal, the end-stage liver disease is, after tuberculosis, the second leading cause of death among HIV-positive people. ${ }^{2}$

Recent studies show, however, some successful treatments for HCV using drug combination in individuals coinfected with HIV. Furthermore, most people with HCV can be treated successfully for HIV. ${ }^{11}$ However, more studies are needed to show the efficacy of new antiviral drugs for HCV in people coinfected with HIV.

In this paper, we study a mathematical model for HIV and HCV coinfection in men who have sex with men (MSM). The model includes treatment for both diseases, screening, awareness and unawareness of HIV infection, and effective protection against HIV and HCV by condom use. We believe this is a relevant study, since $\mathrm{HIV}$ and $\mathrm{HCV}$ are common diseases worldwide and since 2000 outbreaks of acute $\mathrm{HCV}$ among HIV-positive MSM, who denied injecting drugs, have been reported in Europe, ${ }^{12}$ United States ${ }^{13}$ and Australia. ${ }^{14}$ This fact added to the increased severity of the coexistence of the two diseases, makes it extremely important to unravel the dynamics of their coinfection.

HIV and HCV share the same transmission routes, namely by injection drug use (IDU), sexual contact, mother to child transmission during pregnancy or birth, blood and blood products transfusion, organs transplantation from infected donors and exposure to blood by health care professionals. ${ }^{15}$ Here, we will only consider 
the transmission of HIV and HCV by sexual activity. HCV transmission by sexual activity is believed to be inefficient and an accurate estimate for the value of the risk associated to different sexual activities is still to be found. Moreover, the HCV prevalence differs in what concerns the HIV transmission route, being the higher associated to IDU. Nevertheless, there is stronger proof of sexually transmitted HCV in HIV infected MSM. ${ }^{16,17}$ There is also increased concern with the likely bridging of $\mathrm{HCV}$ transmission from the HIV-positive into the HIV-negative MSM. ${ }^{17}$

Bearing these ideas in mind, the paper is organized as follows. The model is described in Sec. 2. In Sec. 2.2, we compute the reproduction numbers and the local stability of the disease-free equilibria. In Sec. 3, we present several bifurcation diagrams that help to understand the dynamical behavior of the proposed model. We compute the sensitivity indices of the reproduction number to relevant parameters of the model in Sec. 4. Simulation results of the full model are presented in Sec. 5. Finally, in Sec. 6, we write the main conclusions of this work.

\section{Model for HIV/AIDS and HCV Transmission}

In this section, we describe the HIV and HCV coinfection model. We compute the reproduction numbers of the full model, and the two submodels (HIV only and HCV only models). We study the local stability of the disease-free equilibria. We compute the sensitivity indices of the reproduction number to relevant parameters of the model. We present bifurcation diagrams, built with the help of XPPAUT, to better understand the dynamics of the proposed model.

\subsection{Description of the model}

The total population, $N(t)$ is divided into the following 16 classes: the unscreened susceptible individuals, $S_{\tilde{s}}(t)$, the screened susceptible individuals, $S_{s}(t)$, the HIV unaware infected individuals, $I_{\tilde{a}}(t)$, the HIV aware infected individuals, $I_{a}(t)$, the unaware individuals showing symptoms of $\operatorname{AIDS}, A_{\tilde{a}}(t)$, the aware individuals that developed AIDS, $A_{a}(t)$, the HCV infected individuals screened for HIV, $I_{c}(t)$, the $\mathrm{HCV}$ infected individuals unscreened for HIV, $I_{\tilde{c}}(t)$, the chronic HCV infected individuals unscreened for HIV, $C_{\tilde{s}}(t)$, the chronic HCV infected individuals screened for HIV, $C_{s}(t)$, the HCV and HIV unaware coinfected individuals, $I_{c} I_{\tilde{a}}(t)$, the HCV and HIV aware coinfected individuals, $I_{c} I_{a}(t)$, the chronic HCV and HIV unaware coinfected individuals, $C I_{\tilde{a}}(t)$, the chronic $\mathrm{HCV}$ and HIV aware coinfected individuals, $C I_{a}(t)$, the $\mathrm{HCV}$ and AIDS dually infected individuals, $I_{c} A(t)$, and the chronic HCV and AIDS coinfected individuals, $C A(t)$.

The schematic diagram of the model is given in Fig. 1.

The population of size $N(t)$, at time $t$, has constant inflow of susceptibles $S_{\tilde{s}}(t)$ and $S_{s}(t), \Lambda=\Lambda_{1}+\Lambda_{2}$. The natural mortality rate is $\mu$ in all classes. Susceptible 


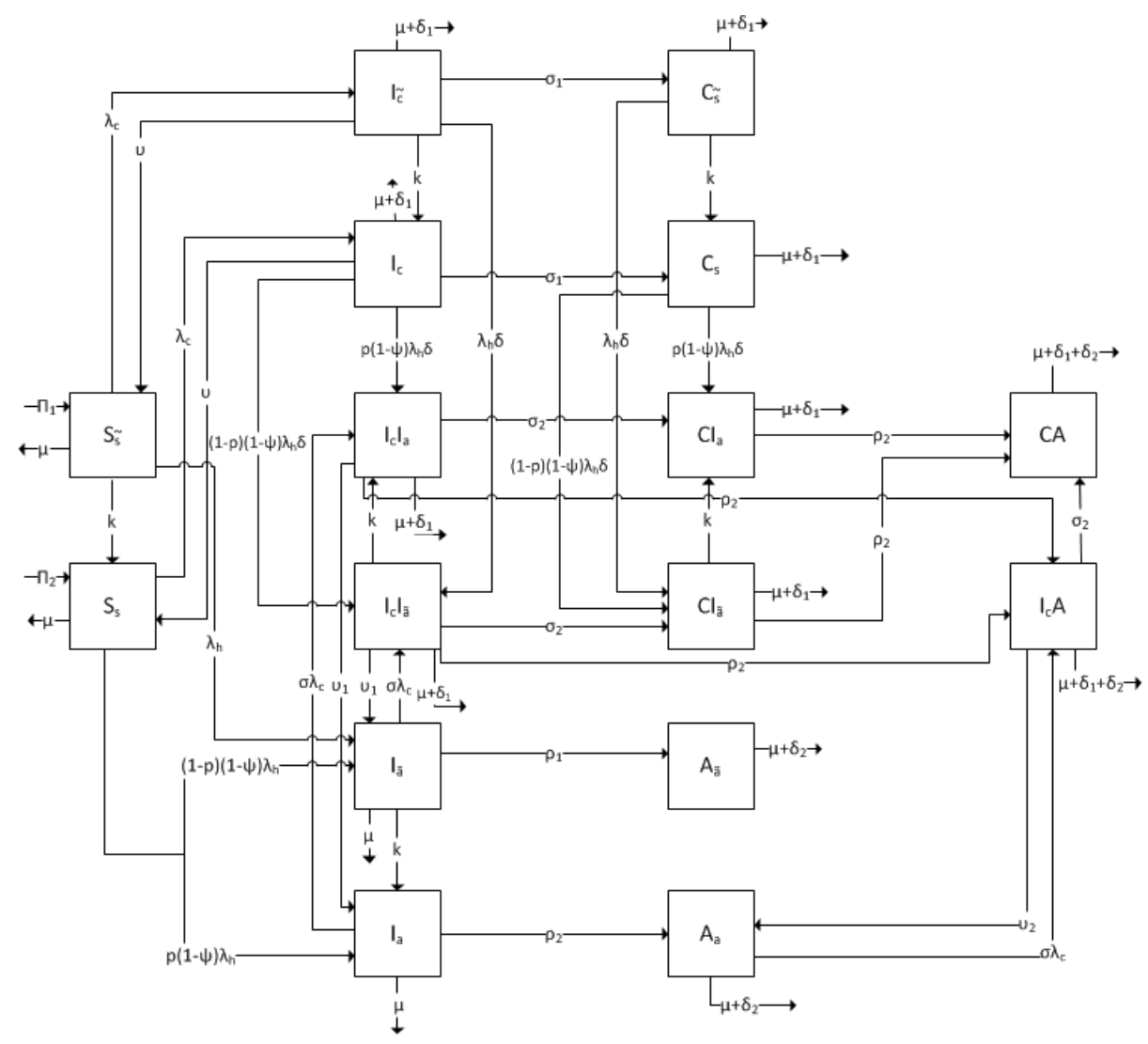

Fig. 1. Flow chart of the model.

unscreened individuals, $S_{\tilde{s}}(t)$, get infected with HIV at a rate $\lambda_{h}$, where:

$$
\lambda_{h}=c(1-\theta) b_{h} \frac{I_{a}(t)+I_{\tilde{a}}(t)+\eta_{1}\left(I_{c} I_{\tilde{a}}(t)+I_{c} I_{a}(t)\right)}{N(t)} .
$$

Parameter $b_{h}$ is the probability that a contact will result in an HIV infection and $c$ is the mean number of sexual partners that a susceptible individual acquires, annually. To model the impact of condom use as a primary prevention tool, we assume that the level of protection by condoms is given by $\theta \in[0,1]$. If $\theta=0$, then condoms do not offer any protection, whereas $\theta=1$ implies perfect protection. ${ }^{6}$ Parameter $\eta_{1}>1$ models the fact that dually infected individuals are more infectious than their corresponding counterparts. ${ }^{18-20}$ Unscreened individuals are screened at a rate $k$ and move to the class of screened susceptible individuals, $S_{s}(t)$.

Screened susceptible individuals, $S_{s}(t)$ are infected with HIV at a rate $(1-\psi) \lambda_{h}$, where $0 \leq \psi \leq 1$ measures the efficacy of screening in reducing disease transmission. If $\psi=0$, then the screening has no effect on the behavior of individuals, on the 
contrary, if $\psi=1$ then screening is $100 \%$ effective in preventing HIV transmission. We consider that screened susceptible individuals can be infected. Routine testing serves only one individual knowing his or her HIV status and accessing care if he or she needs it. A proportion $p$ of screened individuals enter the infected class of individuals who are aware of their condition, $I_{a}(t)$. A proportion $(1-p)$ of infected individuals that are not routinely screened go to the class $I_{\tilde{a}}(t)$ and are unaware of their infectious state. These unscreened individuals are screened after some time at a rate $k$ and moved to class $I_{a}(t)$. Individuals at class $I_{a}(t)$ receive HAART treatment, nevertheless there are some that develop AIDS at a rate $\rho_{2}$. Individuals in class $I_{\tilde{a}}(t)$ may also develop AIDS at a rate $\rho_{1}$. We assume $\rho_{1} \geq \rho_{2}$, since individuals at class $I_{\tilde{a}}(t)$ do not receive HAART treatment, due to their unawareness of the disease condition. The AIDS induced death rate is $\delta_{2}$.

Considering now HCV infection, susceptible unscreened individuals, $S_{\tilde{s}}(t)$ are infected with $\mathrm{HCV}$ at a rate $\lambda_{c}$, and move to class $I_{\tilde{c}}$, where:

$$
\lambda_{c}=c(1-\theta) b_{c} \frac{I_{c}(t)+I_{\tilde{c}}(t)+\eta_{2}\left(I_{c} I_{\tilde{a}}(t)+I_{c} I_{a}(t)\right)}{N(t)} .
$$

Parameter $b_{c}$ is the probability that a contact will result in an HCV infection, $\eta_{2}>1$ models the fact that dually infected individuals are more infectious than their corresponding counterparts. ${ }^{18-20}$ The HCV, unscreened for HIV, infected individuals, $I_{\tilde{c}}(t)$, recover spontaneously (or from treatment), at a rate $v$, and return to class $S_{\tilde{s}}(t)$. They can also progress to a chronic stage, $C_{\tilde{s}}(t)$, at a rate $\sigma_{1}$. Or they can be screened at a rate $k$ and move to class $I_{c}(t)$. These individuals are infected with HIV at a rate $\delta \lambda_{h}$, where $\delta$ accounts for the susceptibility to HIV infection for HCV infected people. ${ }^{20,21}$

The screened susceptible individuals, $S_{s}(t)$ are infected with $\mathrm{HCV}$ at a rate $\lambda_{c}$ and moved to class $I_{c}(t)$. The screened HCV infected individuals are infected with HIV at a rate $(1-\psi) \lambda_{h} \delta$, recover at a rate $v$ and move back to class $S_{s}(t)$, or progress to a chronic phase at a rate $\sigma_{1}$ and move to class $C_{s}(t)$.

The chronic HCV infected individuals, unscreened for HIV, $C_{\tilde{s}(t)}$, are infected with HIV at a rate $\delta \lambda_{h}$ and move to the class $C I_{\tilde{a}}(t)$, and are screened at a rate $k$ and move to class $C_{s}(t)$. The chronic HCV infected individuals, screened for HIV, $C_{s}(t)$, are infected with $\mathrm{HIV}$ at a rate $(1-\psi) \delta \lambda_{h}$.

The HIV unaware infected individuals, $I_{\tilde{a}}(t)$, are infected with $\mathrm{HCV}$ at a rate $\sigma \lambda_{c}$, and move to class $I_{c} I_{\tilde{a}}(t)$, where $\sigma>1$ is the modification parameter modeling the increased risk of being infected with $\mathrm{HCV}$ when a person is already infected with HIV. ${ }^{17,19,20}$ Reciprocally, the HCV infected individuals, screened for HIV, $I_{c}(t)$, are infected with HIV at a rate $(1-p)(1-\psi) \delta \lambda_{h}$ and also move to class $I_{c} I_{\tilde{a}}(t)$. Individuals in class $I_{c} I_{\tilde{a}}(t)$ spontaneously recover from $\mathrm{HCV}$ infection, at a rate $v_{1}$, and move to class $I_{\tilde{a}}(t)$, or progress to AIDS at a rate $\rho_{2}$ and move to class $I_{c} A(t)$. They can also be screened for HIV and move to class $I_{c} I_{a}(t)$. Finally individuals in class $I_{c} I_{\tilde{a}}(t)$ can become chronic carriers at a rate $\sigma_{2}$ and move to class $C I_{\tilde{a}}(t)$. 
The HIV aware infected individuals, $I_{a}(t)$, are infected with HCV at a rate $\sigma \lambda_{c}$, and move to class $I_{c} I_{a}(t)$. Reciprocally, the HCV infected individuals, $I_{c}(t)$, are infected with HIV at a rate $\delta(1-\psi) \lambda_{h}$. Individuals in class $I_{c} I_{a}(t)$ spontaneously recover from $\mathrm{HCV}$ infection at a rate $v_{1}$ and move to the class $I_{a}(t)$. They progress to AIDS at a rate $\rho_{2}$, and move to class $I_{c} A(t)$. Or they may become $\mathrm{HCV}$ chronic carriers, at a rate $\sigma_{2}$, and move to class $C I_{a}(t)$.

The individuals with chronic HCV infection, and unscreened for HIV, $C_{\tilde{s}}(t)$, are infected with HIV at a rate $\delta \lambda_{h}$ and move to class $C I_{\tilde{a}}(t)$. Individuals in this class are screened at a rate $k$ and move to class $C I_{a}$ or progress to AIDS at a rate $\rho_{2}$. The HCV induced death is $\delta_{1}$ in all HCV classes.

The HCV infected individuals at chronic phase, screened for HIV, $C_{s}(t)$, are infected with HIV at a rate $(1-\psi) \delta \lambda_{h}$. The chronic HCV carriers and HIV aware dually infected individuals, $C I_{a}(t)$ progress to AIDS at a rate $\rho_{2}$ and move to class $C A(t)$.

AIDS aware individuals, $A_{s}(t)$, are infected with $\mathrm{HCV}$ at a rate $\sigma \lambda_{c}$ and move to class $I_{c} A(t)$. The individuals in class $I_{c} A(t)$ are treated for $\mathrm{HCV}$ infection at a rate $v_{2}$ and move to class $A_{s}$. The individuals in class $I_{c} A(t)$ become HCV chronic carriers at a rate $\sigma_{2}$ and move to class $C A(t)$.

The parameters and variables of the model are summarized in Table 1.

The system of nonlinear ordinary differential equations for the proposed model is given by:

$$
\begin{aligned}
\frac{d S_{\tilde{s}}}{d t} & =\Lambda_{1}+v I_{\tilde{c}}-\lambda_{h} S_{\tilde{s}}-\lambda_{c} S_{\tilde{s}}-k S_{\tilde{s}}-\mu S_{\tilde{s}} \\
\frac{d S_{s}}{d t} & =\Lambda_{2}+k S_{\tilde{s}}-(1-\psi) \lambda_{h} S_{s}-\lambda_{c} S_{s}+v I_{c}-\mu S_{s} \\
\frac{d I_{\tilde{a}}}{d t} & =(1-p)(1-\psi) \lambda_{h} S_{s}+\lambda_{h} S_{\tilde{s}}+v_{1} I_{c} I_{\tilde{a}}-\left(k+\rho_{1}+\sigma \lambda_{c}+\mu\right) I_{\tilde{a}} \\
\frac{d I_{a}}{d t} & =p(1-\psi) \lambda_{h} S_{s}+k I_{\tilde{a}}+v_{1} I_{c} I_{a}-\left(\sigma \lambda_{c}+\rho_{2}+\mu\right) I_{a} \\
\frac{d A_{\tilde{a}}}{d t} & =\rho_{1} I_{\tilde{a}}-\left(\mu+\delta_{2}\right) A_{\tilde{a}} \\
\frac{d A_{a}}{d t} & =\rho_{2} I_{a}+v_{2} I_{c} A-\left(\sigma \lambda_{c}+\mu+\delta_{2}\right) A_{a} \\
\frac{d I_{\tilde{c}}}{d t} & =\lambda_{c} S_{\tilde{s}}-\left(k+v+\sigma_{1}+\delta \lambda_{h}+\mu+\delta_{1}\right) I_{\tilde{c}} \\
\frac{d I_{c}}{d t} & =\lambda_{c} S_{s}+k I_{\tilde{c}}-\left(v+\sigma_{1}+(1-\psi) \delta \lambda_{h}+\mu+\delta_{1}\right) I_{c} \\
\frac{d C_{\tilde{s}}}{d t} & =\sigma_{1} I_{\tilde{c}}-\left(k+\delta \lambda_{h}+\mu+\delta_{1}\right) C_{\tilde{s}} \\
\frac{d C_{s}}{d t} & =\sigma_{1} I_{c}+k C_{\tilde{s}}-\left((1-\psi) \delta \lambda_{h}+\mu+\delta_{1}\right) C_{s}
\end{aligned}
$$


Table 1. Definition of parameters and variable of model (2.1).

\begin{tabular}{|c|c|}
\hline Variable/Parameter & Description \\
\hline$S_{s}(t)$ & susceptible screened individuals \\
\hline$S_{\tilde{s}}(t)$ & susceptible unscreened individuals \\
\hline$I_{a}(t)$ & HIV infected aware individuals \\
\hline$I_{\tilde{a}}(t)$ & HIV infected unaware individuals \\
\hline$A_{a}(t)$ & aware individuals showing symptoms of AIDS \\
\hline$A_{\tilde{a}}(t)$ & unaware individuals showing symptoms of AIDS \\
\hline$I_{c}(t)$ & screened for HIV, HCV infected individuals \\
\hline$I_{\tilde{c}}(t)$ & unscreened for HIV, HCV infected individuals \\
\hline$C_{s}(t)$ & screened for HIV, chronic HCV infected individuals \\
\hline$C_{\tilde{s}}(t)$ & unscreened for HIV, chronic HCV infected individuals \\
\hline$I_{c} I_{a}(t)$ & HCV and HIV aware coinfected individuals \\
\hline$I_{c} I_{\tilde{a}}(t)$ & HCV and HIV unaware coinfected individuals \\
\hline$C I_{a}(t)$ & HIV aware and chronic HCV dually infected individuals \\
\hline$C I_{\tilde{a}}(t)$ & HIV unaware and chronic HCV coinfected individuals \\
\hline$I_{c} A(t)$ & HCV and AIDS coinfected individuals \\
\hline$C A(t)$ & chronic HCV and AIDS coinfected individuals \\
\hline$\Lambda_{1}$ & recruitment rate of unscreened individuals \\
\hline$\Lambda_{2}$ & recruitment rate of screened individuals \\
\hline$\mu$ & natural mortality rate \\
\hline$b_{h}$ & probability that a contact will result in an HIV infection \\
\hline$b_{c}$ & probability that a contact will result in an $\mathrm{HCV}$ infection \\
\hline$c$ & mean number sexual partners a susceptible individual acquires \\
\hline$\theta$ & level of protection by condoms \\
\hline$p$ & proportion of screened individuals aware of their HIV status \\
\hline$\psi$ & efficacy of screening in reducing HIV transmission \\
\hline$v, v_{i}, i=1,2$ & recovery rate of treated for $\mathrm{HCV}$ \\
\hline$\sigma_{i}, i=1,2$ & rate of progression to chronic phase of $\mathrm{HCV}$ \\
\hline$\delta_{1}$ & mortality due to $\mathrm{HCV}$ \\
\hline$\rho_{i}, i=1,2$ & rate of progression to AIDS \\
\hline$k$ & awareness of unscreened HIV infected individuals after being screened \\
\hline$\delta_{2}$ & mortality due to HIV \\
\hline$\eta_{i}, i=, 1,2$ & modification parameter \\
\hline$\sigma$ & modification parameter \\
\hline$\delta$ & modification parameter \\
\hline
\end{tabular}

$$
\begin{aligned}
\frac{d I_{c} I_{\tilde{a}}}{d t} & =(1-p)(1-\psi) \delta \lambda_{h} I_{c}+\delta \lambda_{h} I_{\tilde{c}}+\sigma \lambda_{c} I_{\tilde{a}}-\left(k+\sigma_{2}+\rho_{2}+v_{1}+\mu+\delta_{1}\right) I_{c} I_{\tilde{a}} \\
\frac{d I_{c} I_{a}}{d t} & =p(1-\psi) \delta \lambda_{h} I_{c}+\sigma \lambda_{c} I_{a}+k I_{c} I_{\tilde{a}}-\left(v_{1}+\rho_{2}+\sigma_{2}+\mu+\delta_{1}\right) I_{c} I_{a} \\
\frac{d C I_{\tilde{a}}}{d t} & =(1-p)(1-\psi) \lambda_{h} \delta C_{s}+\delta \lambda_{h} C_{\tilde{s}}+\sigma_{2} I_{c} I_{\tilde{a}}-\left(k+\rho_{2}+\mu+\delta_{1}\right) C I_{\tilde{a}} \\
\frac{d C I_{a}}{d t} & =p(1-\psi) \delta \lambda_{h} C_{s}+\sigma_{2} I_{c} I_{a}+k C I_{\tilde{a}}-\left(\rho_{2}+\mu+\delta_{1}\right) C I_{a} \\
\frac{d I_{c} A}{d t} & =\sigma \lambda_{c} A_{a}+\rho_{2} I_{c} I_{\tilde{a}}+\rho_{2} I_{c} I_{a}-\left(v_{2}+\sigma_{2}+\mu+\delta_{1}+\delta_{2}\right) I_{c} A \\
\frac{d C A}{d t} & =\rho_{2} C I_{\tilde{a}}+\rho_{2} C I_{a}+\sigma_{2} I_{c} A-\left(\mu+\delta_{1}+\delta_{2}\right) C A
\end{aligned}
$$


with non-negative initial conditions given by:

$$
\begin{aligned}
& S_{s}(0)=S_{s 0}, \quad S_{\tilde{s}}=S_{\tilde{s} 0}, \quad I_{\tilde{a}}(0)=I_{\tilde{a} 0}, \quad I_{a}=I_{a 0}, \quad A_{a}(0)=A_{a 0}, \\
& A_{\tilde{a}}=A_{\tilde{a} 0}, \quad I_{c}(0)=I_{c 0}, \quad I_{\tilde{c}}=I_{\tilde{c} 0}, \quad C_{s}(0)=C_{s 0}, \\
& C_{\tilde{s}}=C_{\tilde{s} 0} I_{c} I_{\tilde{a}}=I_{c} I_{\tilde{a} 0}, \quad I_{c} I_{a}(0)=I_{c} I_{a 0}, \quad C I_{\tilde{a}}(0)=C I_{\tilde{a} 0}, \\
& C I_{a}(0)=C I_{a 0}, \quad I_{c} A(0)=I_{c} A_{0}, \quad C A(0)=C A_{0}
\end{aligned}
$$

Model (2.1) will be analyzed in the domain $\Omega \subset \mathbb{R}_{+0}^{16}$, given by:

$$
\begin{array}{r}
\Omega=\left\{\left(S_{s}, S_{\tilde{s}}, I_{a}, I_{\tilde{a}}, A_{a}, A_{\tilde{a}}, I_{c}, I_{\tilde{c}}, C_{s}, C_{\tilde{s}} m, I_{c} I_{a}, C I_{a}, I_{c} I_{\tilde{a}}, C I_{a},\right.\right. \\
\left.\left.C I_{\tilde{a}}, I_{c} A, C A\right) \in \mathbb{R}_{+}^{16}: 0 \leq N \leq \frac{\Lambda_{1}+\Lambda_{2}}{\mu}\right\}
\end{array}
$$

Theorem 2.1. The solutions of system (2.1) with initial conditions (2.2) satisfy $S_{\tilde{s}}(t) \geq 0, S_{s}(t) \geq 0, I_{\tilde{a}}(t) \geq 0, I_{a}(t) \geq 0, A_{\tilde{a}}(t) \geq 0, A_{a}(t) \geq 0, I_{\tilde{c}}(t) \geq 0$, $I_{c}(t) \geq 0, C_{\tilde{s}}(t) \geq 0, C_{s}(t) \geq 0, I_{c} I_{\tilde{a}}(t) \geq 0, I_{c} I_{a}(t) \geq 0, C I_{\tilde{a}}(t) \geq 0, C I_{a}(t) \geq 0$, $I_{c} A(t) \geq 0, C A(t) \geq 0$ for all $t>0$. The region $\Omega \in \mathbb{R}_{+0}^{16}$ is positively invariant and attracting with respect to system (2.1).

Proof. From the first equation of model (2.1) we have:

$$
\dot{S}_{\tilde{s}} \geq-\left[\lambda_{h}+\lambda_{c}+k+\mu\right] S
$$

thus:

$$
S_{\tilde{s}}(t) \geq S_{\tilde{s}}(0) \mathrm{e}^{\left[-\int_{0}^{t}\left[\lambda_{h}(s)+\lambda_{c}(s)+k+\mu\right] d s\right]}>0 .
$$

Similarly for the second equation of system (2.1):

$$
S_{s} \geq S_{s}(0) e^{\left[-\int_{0}^{t}\left((1-\psi)+\lambda_{c}+\mu\right)\right]}>0
$$

In an analogous fashion, we can easily show that $I_{a}(t), I_{\tilde{a}}, A_{a}(t), A_{\tilde{a}}, I_{c}(t), I_{\tilde{c}}$, $C_{s}(t), C_{\tilde{s}}, I_{c} I_{\tilde{a}}(t), I_{c} I_{a}(t), C I_{\tilde{a}}(t), C I_{a}(t), I_{c} A(t)$ and $C A(t)$ are all positive for all $t>0$. We now show that all feasible solutions are uniformly bounded in $\Omega$. Adding all equations of system (2.1), we obtain:

$$
\begin{aligned}
\dot{N}= & \Lambda_{1}+\Lambda_{2}-\mu N-\delta_{2}\left(A_{a}+A_{\tilde{a}}+I_{c} A+C A\right) \\
& -\delta_{1}\left(I_{c}+I_{\tilde{c}}+C_{s}+C_{\tilde{s}}+I_{c} I_{\tilde{a}}+I_{c} I_{a}+C I_{\tilde{a}}+C I_{a}\right) . \\
\dot{N} \leq & \Lambda_{1}+\Lambda_{2}-\mu N
\end{aligned}
$$

Solving this differential equation, we note that:

$$
0 \leq N \leq \frac{\Lambda}{\mu}+N(0) \mathrm{e}^{-\mu t},
$$

where $\Lambda=\Lambda_{1}+\Lambda_{2}, N(0)$ represents the initial value of the model's variables. Then $0 \leq N \leq \frac{\Lambda}{\mu}$, as $t \rightarrow \infty$. Therefore, $\frac{\Lambda}{\mu}$ is an upper bound of $N$ provided that 
$N(0) \leq \frac{\Lambda}{\mu}$. If $N(0)>\frac{\Lambda}{\mu}$, then $N(t)$ will decrease to this level. Thus, all feasible solutions of the system enter or remain in the region $\Omega$. Hence, the region of biological interest $\Omega$ is positively invariant under the flow induced by system (2.1).

\subsection{Reproduction numbers and stability of disease-free equilibria}

In this subsection, we compute the reproduction number, $R_{0}$, of model $(2.1)$. The basic reproduction number is defined as the number of secondary infections due to a single infection in a completely susceptible population.

We begin by considering the following two sub-models of model (2.1). Model (2.3) is derived from model (2.1) by setting the variables concerning HIV dynamics $\left(I_{\tilde{a}}, I_{a}, A_{\tilde{s}}, A_{s}, I_{c} I_{\tilde{a}}, I_{c} I_{a}, C I_{\tilde{a}}, C I_{a}, I_{c} A\right.$ and $\left.C A\right)$ to zero, and model (2.5) follows from model $(2.1)$ by setting the variables concerning $\mathrm{HCV}$ dynamics $\left(I_{\tilde{c}}, I_{c}\right.$, $C_{\tilde{s}}, C_{s}, I_{c} I_{\tilde{a}}, I_{c} I_{a}, C I_{\tilde{a}}, C I_{a}, I_{c} A$ and $C A$ ) to zero.

We now compute the reproduction number, $R_{\mathrm{HCV}}$, of system $(2.3)$. We use the next generation method. ${ }^{22}$

$$
\begin{aligned}
& \frac{d S_{\tilde{s}}}{d t}=\Lambda_{1}+v I_{\tilde{c}}-\lambda_{c} S_{\tilde{s}}-k S_{\tilde{s}}-\mu S_{\tilde{s}}, \quad \frac{d S}{d t}=\Lambda_{2}+k S_{\tilde{s}}-\lambda_{c} S_{s}+v I_{c}-\mu S_{s}, \\
& \frac{d I_{\tilde{c}}}{d t}=\lambda_{c} S_{\tilde{s}}-\left(k+v+\sigma_{1}+\mu+\delta_{1}\right) I_{\tilde{c}}, \quad \frac{d I_{c}}{d t}=\lambda_{c} S_{s}-\left(v+\sigma_{1}+\mu+\delta_{1}\right) I_{c}, \\
& \frac{d C_{\tilde{s}}}{d t}=\sigma_{1} I_{\tilde{c}}-\left(k+\mu+\delta_{1}\right) C_{\tilde{s}}, \quad \frac{d C_{s}}{d t}=\sigma_{1} I_{c}+k C_{\tilde{s}}-\left(\mu+\delta_{1}\right) C_{s},
\end{aligned}
$$

where $\lambda_{c}=c(1-\theta) b_{c} \frac{I_{c}+I_{\tilde{c}}}{N}$.

The disease-free equilibrium of model (2.3) is given by:

$$
P_{0}^{1}=\left(\frac{\Lambda_{1}}{k+\mu}, \frac{\Lambda_{2}}{\mu}+\frac{k \Lambda_{1}}{\mu(k+\mu)}, 0,0,0,0\right) .
$$

Using the notation of Ref. 22, matrices for the new infection terms, $F$, and the other terms, $V$, are given by:

$$
\begin{gathered}
F=\left[\begin{array}{cccc}
c(1-\theta) b_{c} \frac{\Lambda_{1} \mu}{\Lambda_{1}(\mu+1)+\Lambda_{2}(k+\mu)} & c(1-\theta) b_{c} \frac{\Lambda_{1} \mu}{\Lambda_{1}(\mu+1)+\Lambda_{2}(k+\mu)} & 0 & 0 \\
c(1-\theta) b_{c} \frac{\Lambda_{1} \mu+\Lambda_{2}(k+\mu)}{\Lambda_{1}(\mu+1)+\Lambda_{2}(k+\mu)} & c(1-\theta) b_{c} \frac{\Lambda_{1} \mu+\Lambda_{2}(k+\mu)}{\Lambda_{1}(\mu+1)+\Lambda_{2}(k+\mu)} & 0 & 0 \\
0 & 0 & 0 & 0 \\
0 & 0 & 0 & 0
\end{array}\right], \\
V=\left[\begin{array}{cccc}
k+v+\sigma_{1}+\mu+\delta_{1} & 0 & 0 & 0 \\
-k & v+\sigma_{1}+\mu+\delta_{1} & 0 & 0 \\
-\sigma_{1} & 0 & k+\mu+\delta_{1} & 0 \\
0 & -\sigma_{1} & -k & \mu+\delta_{1}
\end{array}\right] .
\end{gathered}
$$


The associative basic reproduction number is thus:

$$
R_{\mathrm{HCV}}=\rho\left(F V^{-1}\right)=\frac{c(1-\theta) b_{c}}{v+\sigma_{1}+\mu+\delta_{1}},
$$

where $\rho$ indicates the spectral radius of $F V^{-1}$. By Theorem $2,{ }^{22}$ we obtain the following lemma.

Lemma 2.1. The disease-free equilibrium $P_{0}^{1}$ is locally asymptotically stable if $R_{\mathrm{HCV}}<1$ and unstable if $R_{\mathrm{HCV}}>1$.

We proceed with the computation of the reproduction number, $R_{\mathrm{HIV}}$, of model (2.5).

$$
\begin{aligned}
\frac{d S_{\tilde{s}}}{d t} & =\Lambda_{1}-\lambda_{h} S_{\tilde{s}}-k S_{\tilde{s}}-\mu S_{\tilde{s}}, \quad \frac{d S_{s}}{d t}=\Lambda_{2}+k S_{\tilde{s}}-(1-\psi) \lambda_{h} S_{s}-\mu S_{s}, \\
\frac{d I_{\tilde{a}}}{d t} & =(1-p)(1-\psi) \lambda_{h} S_{s}+\lambda_{h} S_{\tilde{s}}-\left(k+\rho_{1}+\mu\right) I_{\tilde{a}}, \\
\frac{d I_{a}}{d t} & =p(1-\psi) \lambda_{h} S_{s}+k I_{\tilde{a}}-\left(\rho_{2}+\mu\right) I_{k}, \\
\frac{d A_{\tilde{s}}}{d t} & =\rho_{1} I_{\tilde{a}}-\left(\mu+\delta_{2}\right) A_{\tilde{a}}, \quad \frac{d A}{d t}=\rho_{2} I_{a}-\left(\mu+\delta_{2}\right) A,
\end{aligned}
$$

where $\lambda_{h}=c(1-\theta) b_{h} \frac{I_{\tilde{a}}+I_{a}}{N}$.

The disease-free equilibrium state $P_{0}^{2}$ of model (2.5) is given by:

$$
P_{0}^{2}=\left(\frac{\Lambda_{1}}{k+\mu}, \frac{\Lambda_{2}}{\mu}+\frac{k \Lambda_{1}}{\mu(k+\mu)}, 0,0,0,0\right)
$$

Using the notation of Ref. 22, matrices for the new infection terms, $F$, and the other terms, $V$, are the following:

$$
\begin{aligned}
& F=\left[\begin{array}{c}
\frac{c(1-\theta) b_{h}}{\left(\Lambda_{1}+\Lambda_{2}\right)(\mu+k)}\left[(1-p)(1-\psi)\left(k \Lambda_{1}+\Lambda_{2}(k+\mu)\right)+\Lambda_{1} \mu\right] \\
p(1-\psi) c(1-\theta) b_{h} \frac{k \Lambda_{1}+\Lambda_{2}(k+\mu)}{\left(\Lambda_{1}+\Lambda_{2}\right)(\mu+k)} \\
0 \\
0
\end{array}\right. \\
& \left.\begin{array}{ccc}
\frac{c(1-\theta) b_{h}}{\left(\Lambda_{1}+\Lambda_{2}\right)(\mu+k)}\left[(1-p)(1-\psi)\left(k \Lambda_{1}+\Lambda_{2}(k+\mu)\right)+\Lambda_{1} \mu\right] & 0 & 0 \\
p(1-\psi) c(1-\theta) b_{h} \frac{k \Lambda_{1}+\Lambda_{2}(k+\mu)}{\left(\Lambda_{1}+\Lambda_{2}\right)(\mu+k)} & 0 & 0 \\
0 & 0 & 0 \\
0 & 0 & 0
\end{array}\right], \\
& V=\left[\begin{array}{cccc}
k+\rho_{1}+\mu & 0 & 0 & 0 \\
-k & \rho_{2}+\mu & 0 & 0 \\
-\rho_{1} & 0 & \mu+\delta_{2} & 0 \\
0 & -\rho_{2} & 0 & \mu+\delta_{2}
\end{array}\right]
\end{aligned}
$$


The associative basic reproduction number is given by:

$$
\begin{aligned}
R_{\mathrm{HIV}}= & \rho\left(F V^{-1}\right) \\
= & \frac{c(1-\theta) b_{h}}{\left(k+\mu+\rho_{1}\right)\left(\rho_{2}+\mu\right)\left(\Lambda_{1}+\Lambda_{2}\right)(\mu+k)}\left[(1-\psi)\left(k \Lambda_{1}+\Lambda_{2}(k+\mu)\right)\right. \\
& \left.\times\left(k+\mu+\rho_{2}+p\left(\rho_{1}-\rho_{2}\right)\right)+\Lambda_{1} \mu\left(k+\mu+\rho_{2}\right)\right],
\end{aligned}
$$

where $\rho$ indicates the spectral radius of $F V^{-1}$. By Theorem $2,{ }^{22}$ we obtain the following lemma.

Lemma 2.2. The disease-free equilibrium $P_{0}^{2}$ is locally asymptotically stable if $R_{\mathrm{HIV}}<1$ and unstable if $R_{\mathrm{HIV}}>1$.

We now proceed with the calculation of the reproduction number, $R_{0}$, of the full model (2.1). The disease-free equilibrium state $P_{0}$ of model (2.1) is given by:

$$
P_{0}=\left(\frac{\Lambda_{1}}{k+\mu}, \frac{\Lambda_{2}}{\mu}+\frac{k \Lambda_{1}}{\mu(k+\mu)}, 0,0,0,0,0,0,0,0,0,0,0,0,0,0\right) .
$$

Using the notation in Ref. 22 on system (2.1), we compute the matrices for the new infection terms, $F$, and the other terms, $V$, and the marix $F V^{-1}$. Then, the reproduction number is the spectral radius of $F V^{-1}$. After some algebra manipulation $R_{0}$ is given by (please check the Appendix for a detailed proof):

$$
R_{0}=\rho\left(F V^{-1}\right)=\max \left\{R_{\mathrm{HIV}}, R_{\mathrm{HCV}}\right\}
$$

By Theorem $2,{ }^{22}$ we obtain the following lemma.

Lemma 2.3. The disease-free equilibrium $P_{0}$ is locally asymptotically stable if $R_{0}<1$ and unstable if $R_{0}>1$.

\section{Bifurcation Analysis}

In this section, we use XPPAUT ${ }^{23}$ to build bifurcation diagrams for distinct parameters of model (2.1).

Figure 2 shows the sketch of the bifurcation diagram for different values of $\beta_{c}$, the probability that a contact will result in an $\mathrm{HCV}$ infection. We start from a disease-free equilibrium and increase $\beta_{c}$. At $\beta_{c}=0.2031$, there is a bifurcation point (1), at which the model bifurcates to the stable HCV endemic equilibrium. This means that increasing the probability that a contact will result in an HCV infection originates new cases of HCV infections. This is a realistic prediction.

Figure 3 depicts the bifurcation diagram for different values of $\beta_{h}$, the probability that a contact will result in an HIV infection. We start from a disease-free equilibrium and increase $\beta_{h}$. At $\beta_{h}=0.034$, there is a bifurcation point (1), at which the model bifurcates to the stable HIV endemic equilibrium. This means that increasing the probability that a contact will result in an HIV infection promotes the appearance of new cases of HIV infections, as biologically expected. 


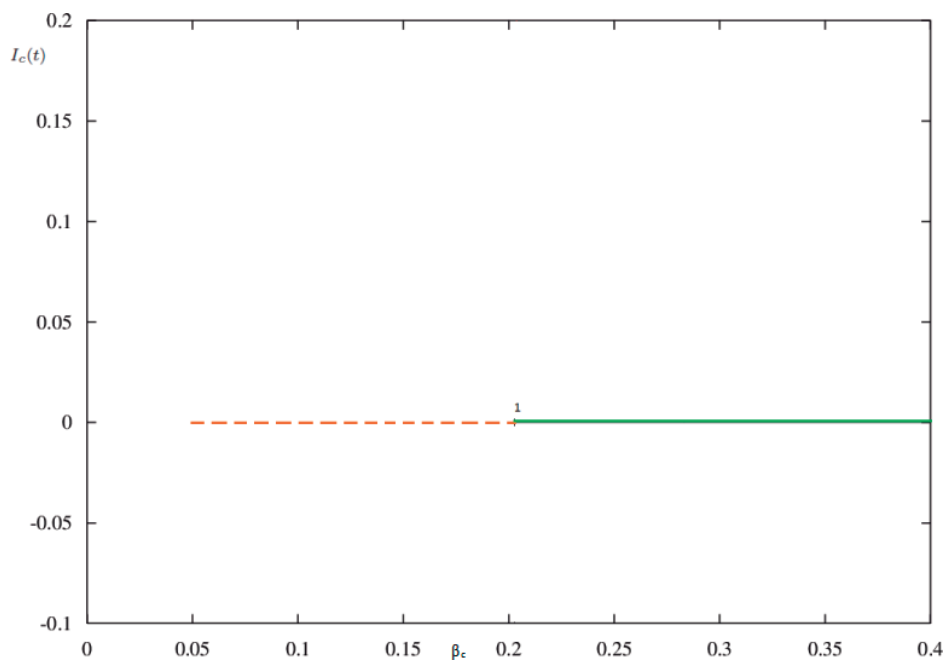

Fig. 2. Sketch of the bifurcation diagram for different values of $\beta_{c}$, the probability that a contact will result in HCV transmission. Remaining parameter values are given in Table 3, except for $\beta_{h}=0.032$. At the bifurcation point (1) $\beta_{c} \simeq 0.2031$. Orange dashed line - stable disease-free equilibrium, green filled line — stable HCV endemic equilibrium. For more information, see text.

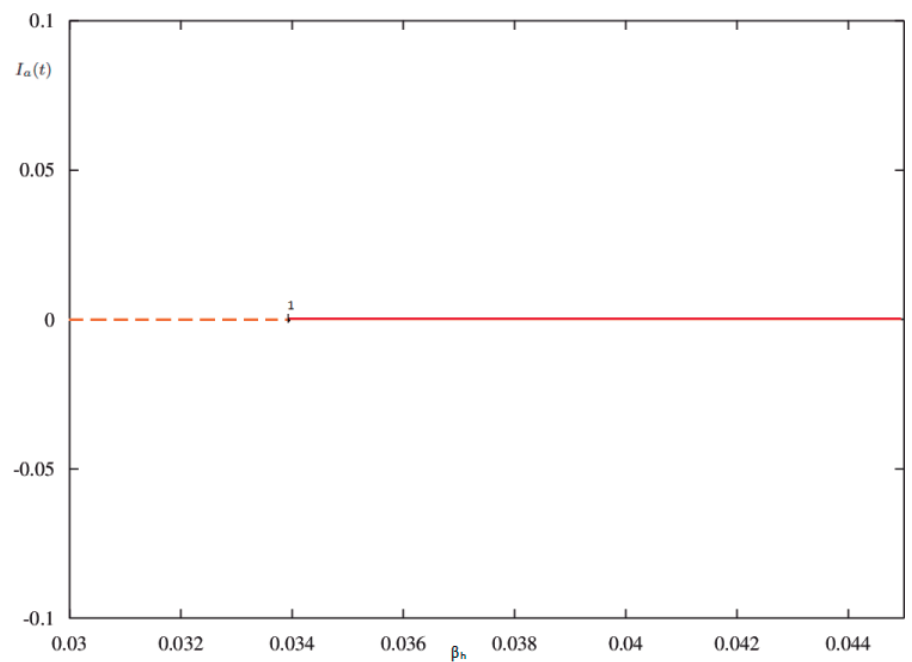

Fig. 3. Drawing of the bifurcation diagram for different values of $\beta_{h}$, the probability that a contact will result in HIV transmission. Remaining parameter values are given in Table 3 . At the bifurcation point (1) $\beta_{h} \simeq 0.034$. Orange dashed line — stable disease-free equilibrium, red filled line — stable HIV endemic equilibrium. For more information, see text.

Figure 4 depicts the bifurcation diagram for different values of $\beta_{c}$, the probability that a contact will result in an HCV infection. We start from an HIV endemic equilibrium and increase $\beta_{c}$. At $\beta_{c}=0.2503$, there is a bifurcation point (1), at which the model bifurcates to the stable two endemic equilibrium. This means 


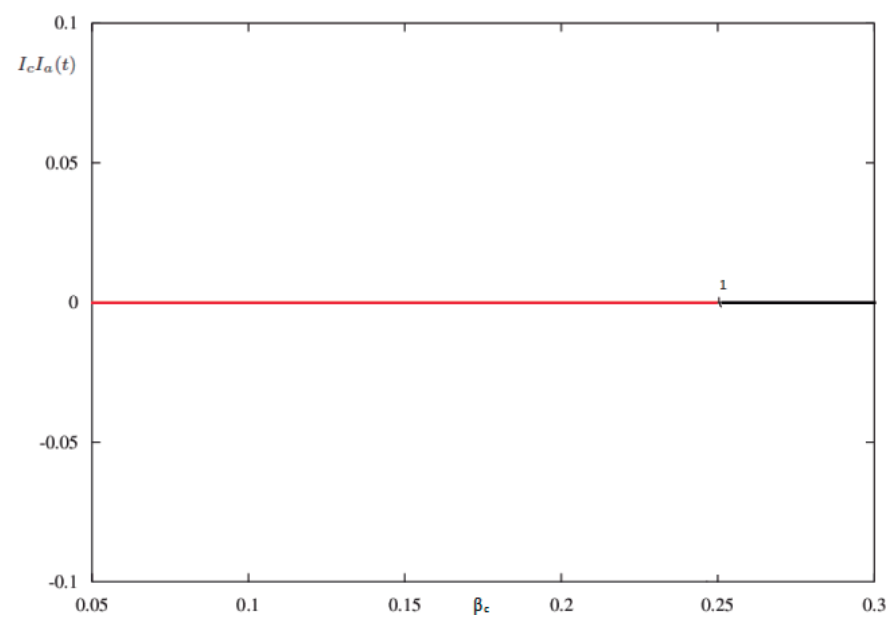

Fig. 4. Sketch of the bifurcation diagram for different values of $\beta_{c}$, the probability that a contact will result in an HCV infection. Remaining parameter values are given in Table 3. At the bifurcation point (1) $\beta_{c}=0.2503$. Red filled line - stable HIV endemic equilibrium, black filled line stable two-endemic equilibrium. For more information, see text.

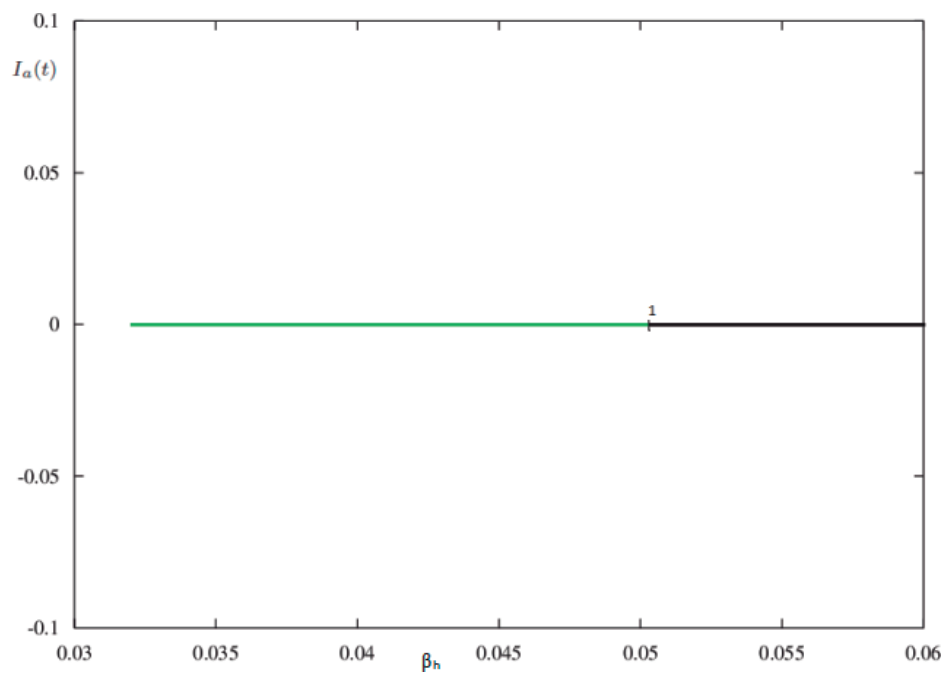

Fig. 5. Sketch of the bifurcation diagram for different values of $\beta_{h}$, the probability that a contact will result in an HIV infection. Remaining parameter values are given in Table 3. At the bifurcation point (1) $\beta_{h}=0.0503$. Green filled line - stable HCV endemic equilibrium, black filled line stable two-endemic equilibrium. For more information, see text.

that in a population already infected with HIV, with lowered defense mechanisms, increasing the probability of a contact resulting in $\mathrm{HCV}$ infection will set off new coinfection cases.

Figure 5 depicts the bifurcation diagram for different values of $\beta_{h}$, the probability that a contact will result in an HIV infection. We start from a HCV endemic 
equilibrium and increase $\beta_{h}$. At $\beta_{h}=0.0503$, there is a bifurcation point (1), at which the model bifurcates to the stable two-endemic equilibrium. This means that in a population with impaired immune system due to infection with HCV, increasing the probability of a contact resulting in HIV infection will trigger new coinfection cases.

\section{Sensitivity Analysis}

In this section, we perform the sensitivity indexes of the reproduction number, $R_{0}$, to relevant parameters of model (2.1). We follow the procedure developed in Ref. 24 . The sensitivity analysis is used to measure the relative change in a state variable when a given parameter is varied. Measuring the $R_{0}$ sensitivity indices is extremely important since the value of $R_{0}$ determines the spread or the eradication of the disease.

The sensitivity index of $R_{0}=\max \left\{R_{\mathrm{HIV}}, R_{\mathrm{HCV}}\right\}$ with respect to parameter $\beta_{c}=c(1-\theta) b_{c}$ is given by:

$$
\frac{\partial R_{0}}{\partial \beta_{c}} \times \frac{\beta_{c}}{R_{0}}=1 .
$$

The sensitivity index of $R_{0}$ with respect to parameter $\beta_{h}=c(1-\theta) b_{h}$ is given by:

$$
\frac{\partial R_{0}}{\partial \beta_{h}} \times \frac{\beta_{h}}{R_{0}}=1
$$

These values for this sensitivity index mean that the probability of HCV or HIV transmission has strong influence in HCV and HIV control and management. Similar expressions may be obtained for the other parameters of $R_{0}$. Nevertheless, most of the expressions for the sensitivity indices are complex with little obvious structure. Therefore, we compute the corresponding values for the parameter values given in Table 3. The signs of the sensitivity indices may be found in Table 2. A positive sign implies that increases in the corresponding parameter values translate in an increase in the value of $R_{0}$. Inversely for a negative sign, a negative index

Table 2. Parameters used in the numerical simulations of model (2.1).

\begin{tabular}{cc}
\hline Parameter & Sensitivity index sign \\
\hline$\beta_{c}$ & + \\
$\beta_{h}$ & + \\
$\theta$ & - \\
$\nu$ & - \\
$\sigma_{1}$ & - \\
$\sigma_{2}$ & - \\
$p$ & + \\
$k$ & + \\
$\rho_{2}$ & - \\
$\rho_{1}$ & - \\
\hline
\end{tabular}


Table 3. Parameters used in the numerical simulations of model (2.1), where the appropriate units are $\mathrm{yr}^{-1}$.

\begin{tabular}{clc}
\hline Parameter & Value & Reference \\
\hline$\Lambda_{1}$ & 8 & Assume \\
$\Lambda_{2}$ & 4 & Assume \\
$c$ & 8 & Assume \\
$\theta$ & 0.20 & 25 \\
$b_{h}$ & 0.036 & 26 \\
$b_{c}$ & 0.05 & 17 \\
$\eta_{1}$ & 1.0002 & 20 \\
$\eta_{2}$ & 1.0002 & 20 \\
$v$ & 0.25 & 17 \\
$v_{1}$ & 0.27 & Assume \\
$v_{2}$ & 0.25 & Assume \\
$\mu$ & 0.020 & 20 \\
$p$ & 0.0367 & 27 \\
$\psi$ & 0.0288 & 27 \\
$k$ & 0.00388 & 27 \\
$\rho_{1}$ & 0.1908 & 27 \\
$\rho_{2}$ & 0.1511 & 27 \\
$\delta_{1}$ & 0.2801 & Assume \\
$\delta_{2}$ & 0.2801 & 27 \\
$\delta$ & 1.0001 & 20 \\
$\sigma$ & 1.001 & 20 \\
$\sigma_{1}$ & 0.75 & 28 \\
$\sigma_{2}$ & 0.75 & 28 \\
\hline
\end{tabular}

means than an increase in the parameter value implies a decrease in the value of $R_{0}$. For example, an increase of $5 \%$ in the values of $\beta_{h}$ or $\beta_{c}$ augments $5 \%$ the value of $R_{0}$. Similar inferences may be performed for the other indices.

\section{Numerical Results}

In this section, we present the numerical simulations of model (2.1). The parameter values used in the simulations can be found in Table 3 and the following initial conditions: $S_{\tilde{s}}(0)=500, S_{s}(t)=0$ (we assume that no individuals were screened initially), $I_{\tilde{a}}(0)=80, I_{a}(0)=0, A_{a}(t)=0, A_{\tilde{a}}=15, I_{c}(t)=0, I_{\tilde{c}}=20, C_{s}(0)=0$, $C_{\tilde{s}}=5, I_{c} I_{a}(0)=I_{c} I_{\tilde{a}}=C I_{a}=C I_{\tilde{a}}=I_{c} A=C A=0$ are used.

In Fig. 6, we observe that the model approaches asymptotically the stable HIV endemic equilibrium.

Figure 7 shows the stable HCV endemic equilibrium for system (2.1).

In Fig. 8, we plot the dynamics of the variables of system (2.1). We observe that, for the given parameters values and initial conditions, the model approaches asymptotically the stable two-disease endemic equilibrium.

In Fig. 9, we plot the dynamics of the relevant variables of system (2.1) for different values of $\theta$, the level of protection against HIV and HCV by the use of condoms. We observe that as $\theta$ decreases, the system (2.1) bifurcates from the stable 

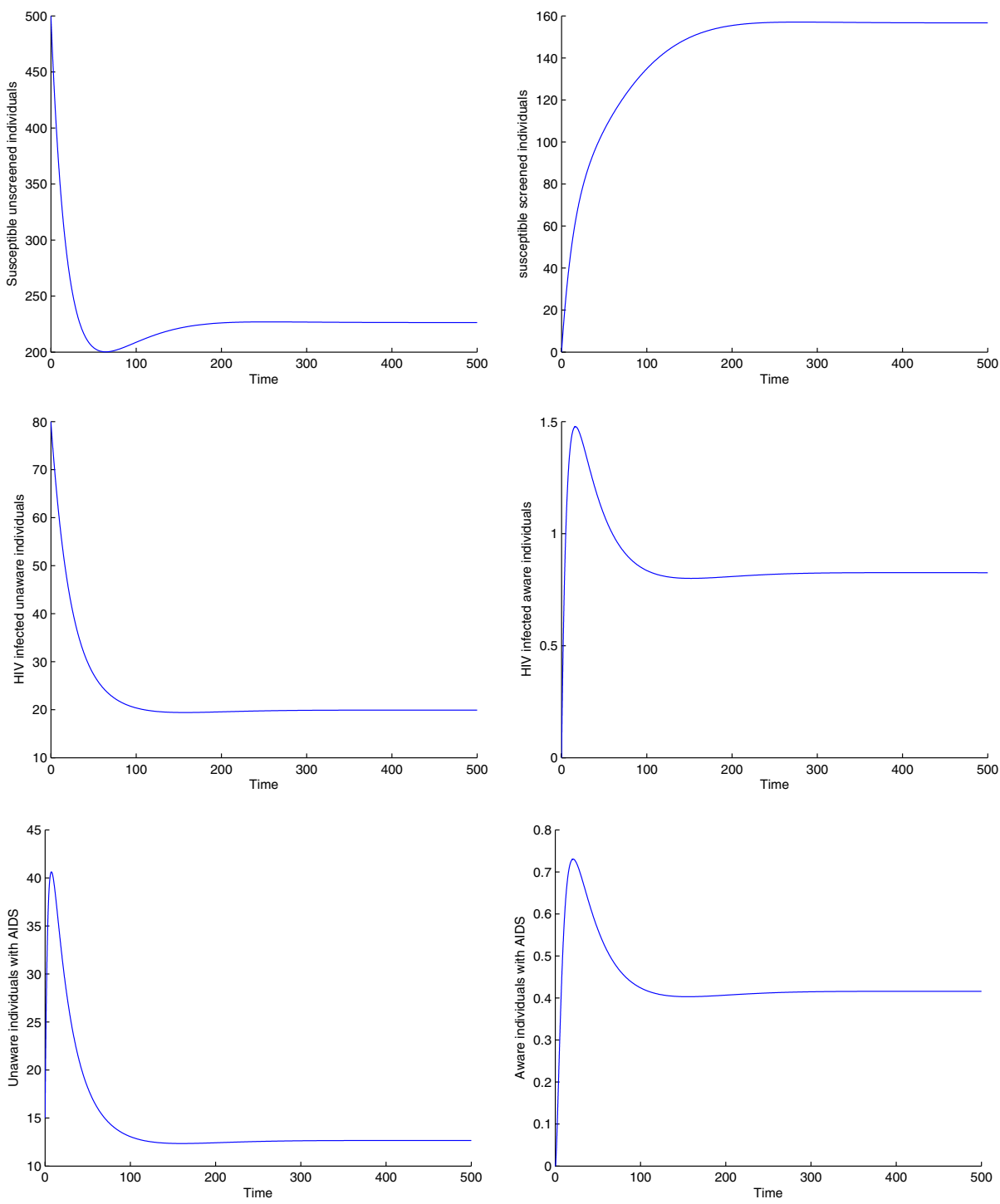

Fig. 6. Stable HIV endemic equilibrium of system (2.1) for given parameter values in Table 3, and given initial conditions $\left(R_{\mathrm{HIV}}=1.0875, R_{\mathrm{HCV}}=0.2461, R_{0}=1.0875\right)$. The remaining variables go asymptotically to 0 . For more information, see text.

disease-free equilibrium to the stable HIV endemic equilibrium. This suggests that individuals should, as expected, use condom, to prevent being infected with HIV.

In Fig. 10, we show the dynamics of the relevant variables of system (2.1) for different values of $\beta_{h}$, the probability that a contact will result in an HIV infection. We observe that increasing the system (2.1) has a bifurcation point as $\beta_{h}$ increases from 0.030 till 0.1 . The system bifurcates from a stable disease-free equilibrium to 

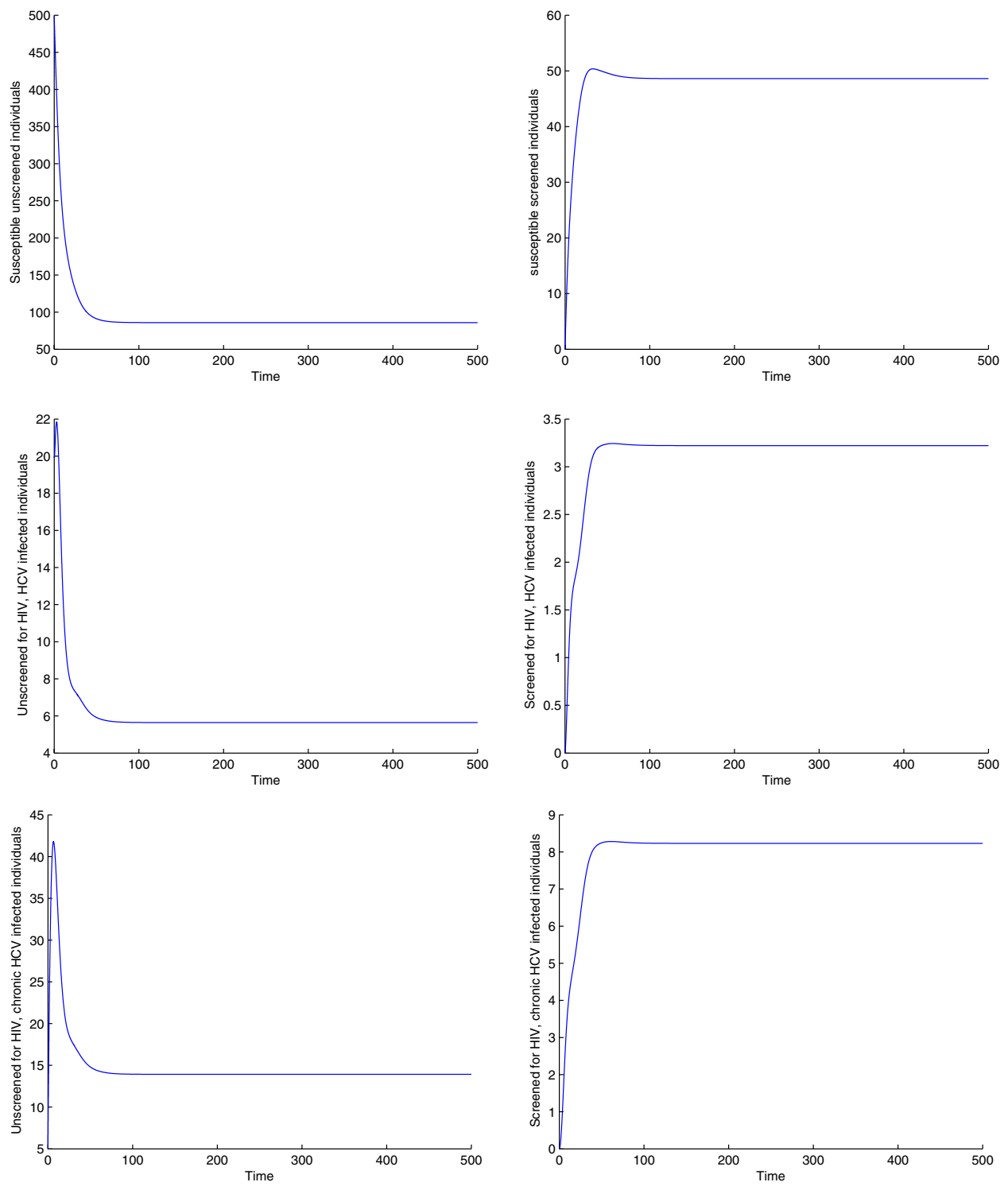

Fig. 7. Stable HCV endemic equilibrium of system (2.1) for parameter values given in Table 3, except $\beta_{c}=0.25$ and $\beta_{h}=0.032$, and given initial conditions $\left(R_{\mathrm{HIV}}=0.9667, R_{\mathrm{HCV}}=1.2307\right.$, $\left.R_{0}=1.2307\right)$. The remaining variables go asymptotically to 0 . For more information, see text.

a stable HIV endemic equilibrium. This was a predictable outcome and suggests the measures that reduce this probability $\beta_{h}$ should be taken under consideration in order to reduce HIV transmission.

In Fig. 11, we plot the dynamics of the relevant variables of system (2.1) for different values of $\beta_{c}$, the probability that a contact will result in an HCV infection. 

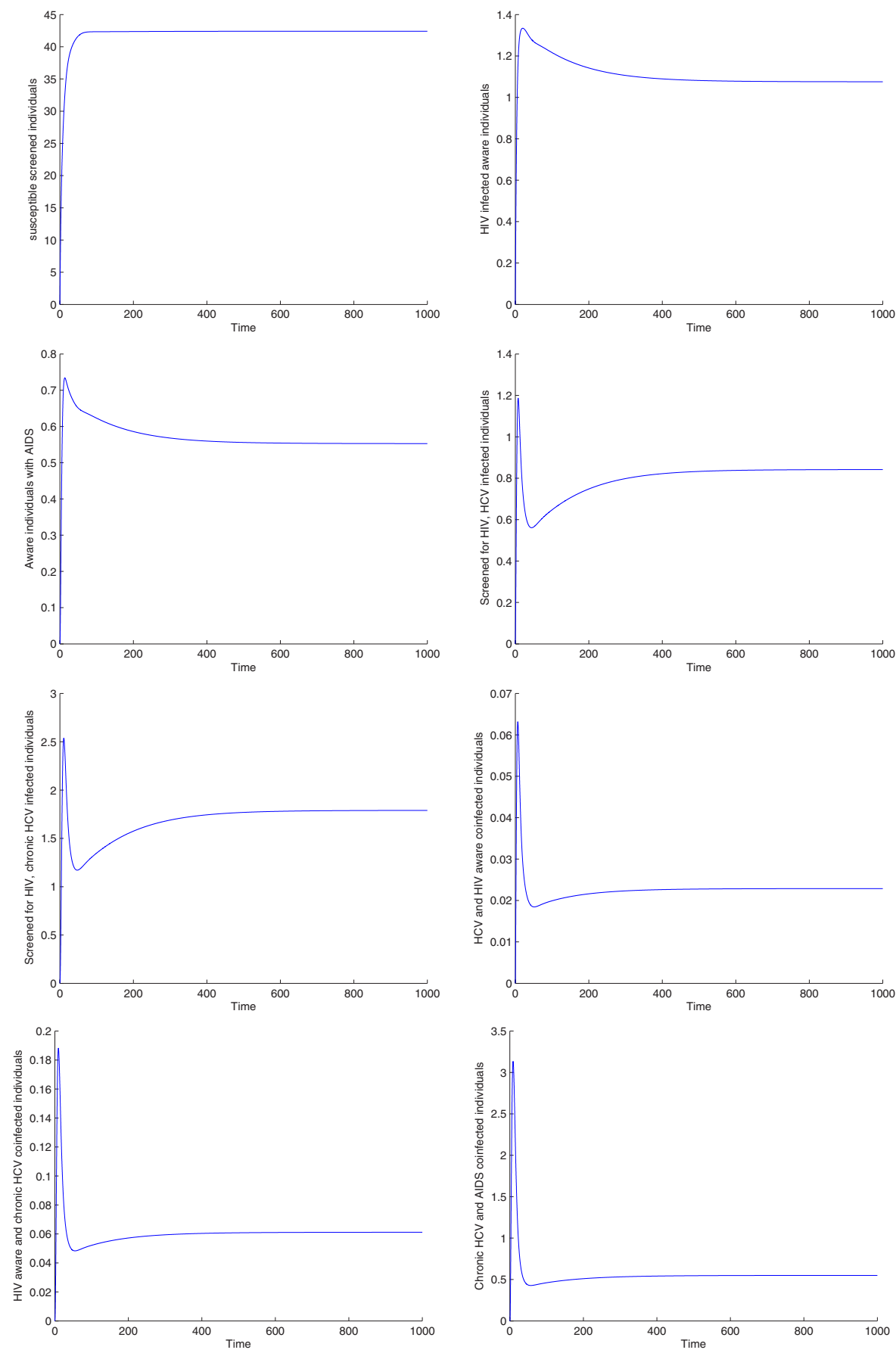

Fig. 8. Stable two-disease endemic equilibrium of system (2.1) for given parameter values in Table 3 , except for $b_{h}=0.054, b_{c}=0.25$, and given initial conditions $\left(R_{\mathrm{HIV}}=1.6313, R_{\mathrm{HCV}}=\right.$ $1.2307, R_{0}=1.6313$ ) (relevant variables). For more information, see text. 

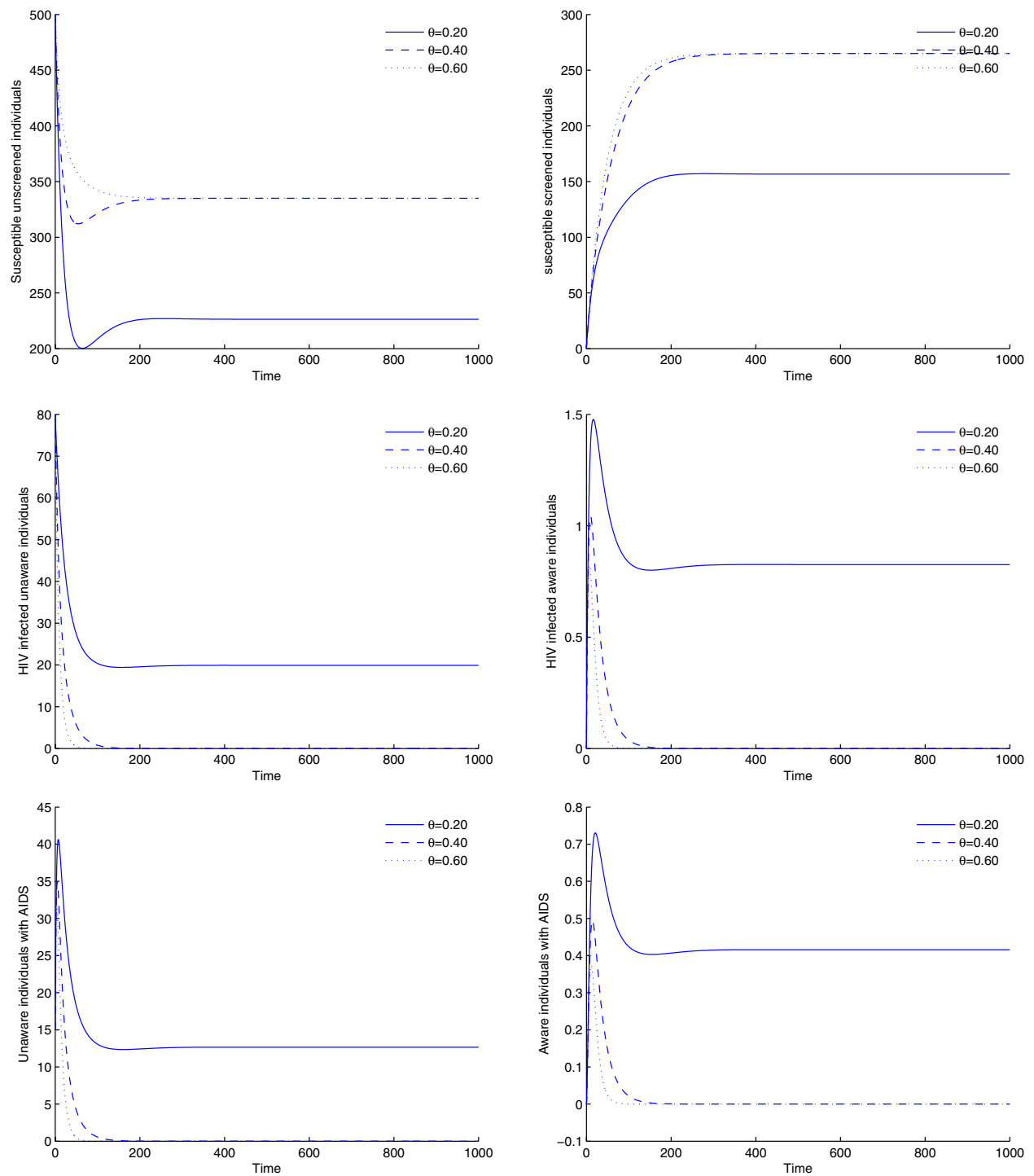

Fig. 9. Dynamics of the relevant variables of the system (2.1) for different values of $\theta$, the level of protection against HIV and HCV by condom use. Parameter values are given in Table 3 and initial conditions are in the text. The remaining variables go asymptotically to zero. For more information, see text.

We observe that as $\beta_{c}$ increases from 0.05 to 0.25 there is a bifurcation from a state without disease to a state with HCV infection that persists with the continuous increase of $\beta_{c}$. The number of chronic carriers also increases. Biologically this is a reasonable outcome, one would expect an augment in the number of new disease cases as the probability of transmission increases. 

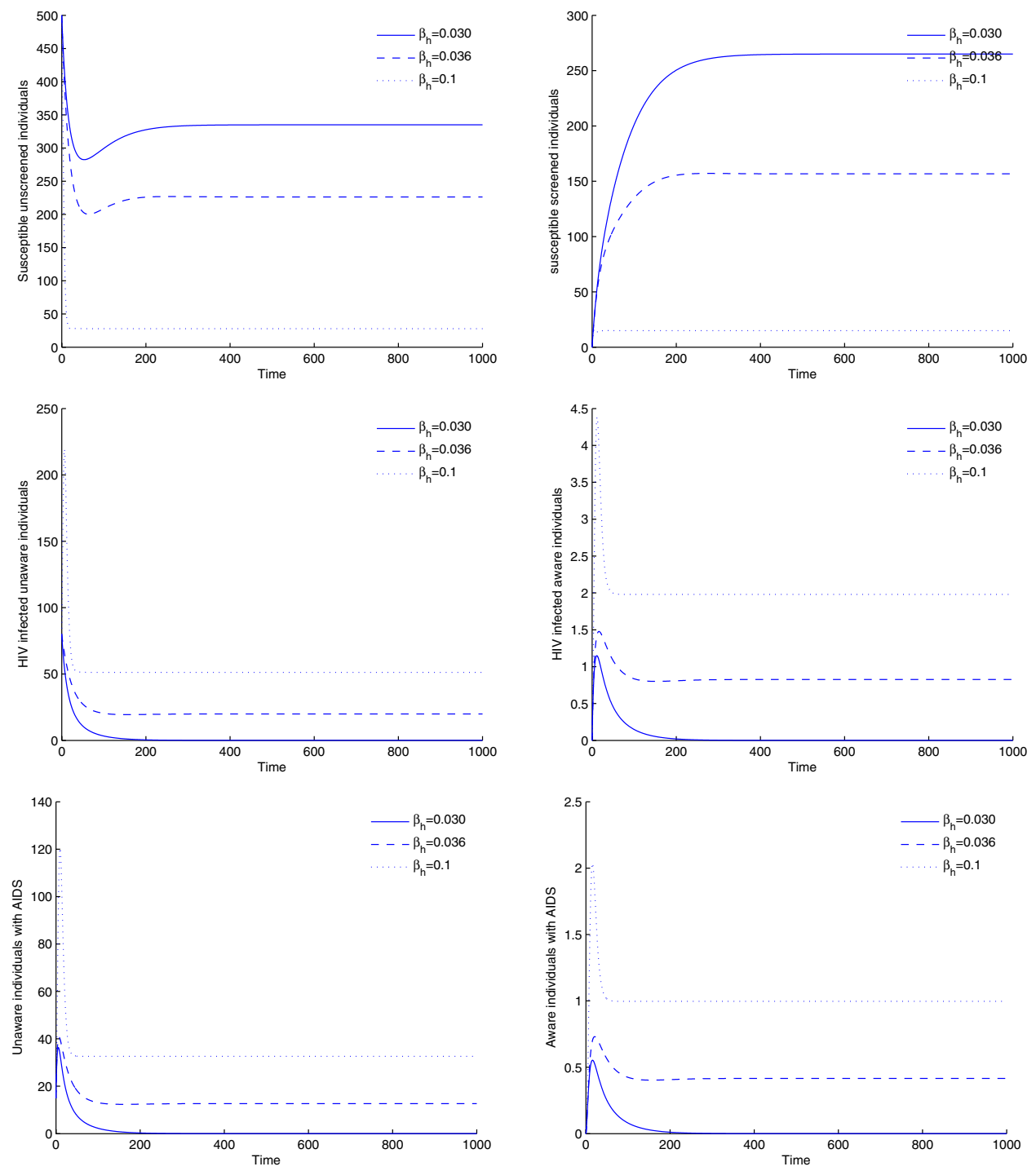

Fig. 10. Dynamics of the relevant variables of the system (2.1) for different values $\beta_{h}$, the probability that a contact will result in an HIV infection. Parameter values are given in Table 3 and initial conditions are in the text. The remaining variables go asymptotically to zero. For more information see text.

In Fig. 12, we plot the dynamics of the relevant variables of system (2.1) for different values of $\psi$, the parameter that measures the efficacy of screening in reducing HIV transmission. We observe that as $\psi$ increases, the number of individuals infected with HIV and suffering from AIDS decreases, in fact, the system (2.1) is initially at a stable HIV endemic equilibrium and changes its qualitative behavior 

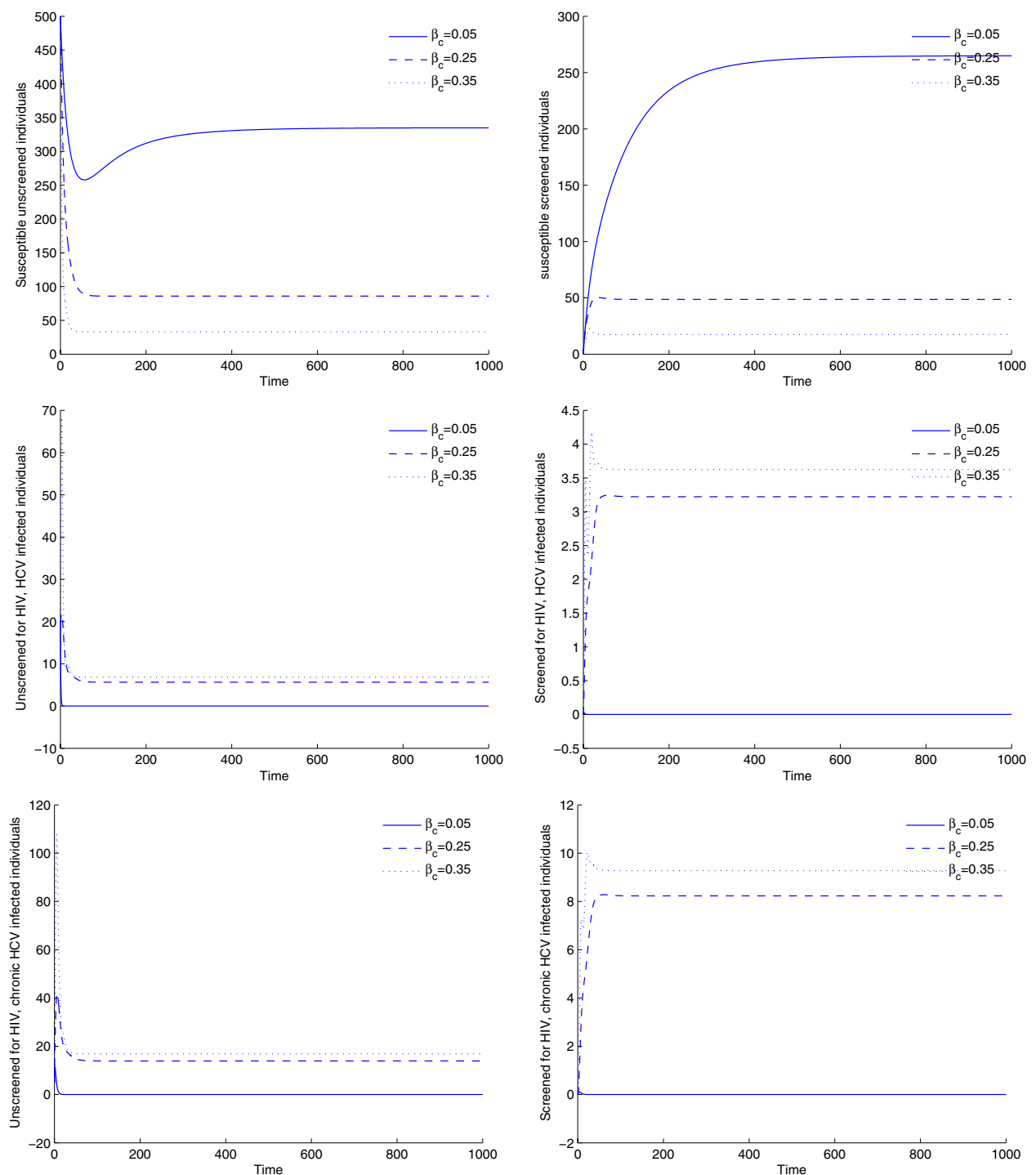

Fig. 11. Dynamics of the relevant variables of the system (2.1) for different values of $\beta_{c}$, the probability that a contact will result in an HCV infection. Parameter values are given in Table 3 , except $b_{h}=0.032$ and initial conditions are in the text. For more information, see text.

to a stable disease-free equilibrium. In real life, this means that individuals that are screened should change their behavior in order to prevent transmission of the disease. In fact, there are some studies that show that screened HIV infectives tend to change their behavior and those whose test is negative do not. ${ }^{29}$ Nevertheless, the tests are meant to promote behavior change and to access to health care for all screened individuals. 

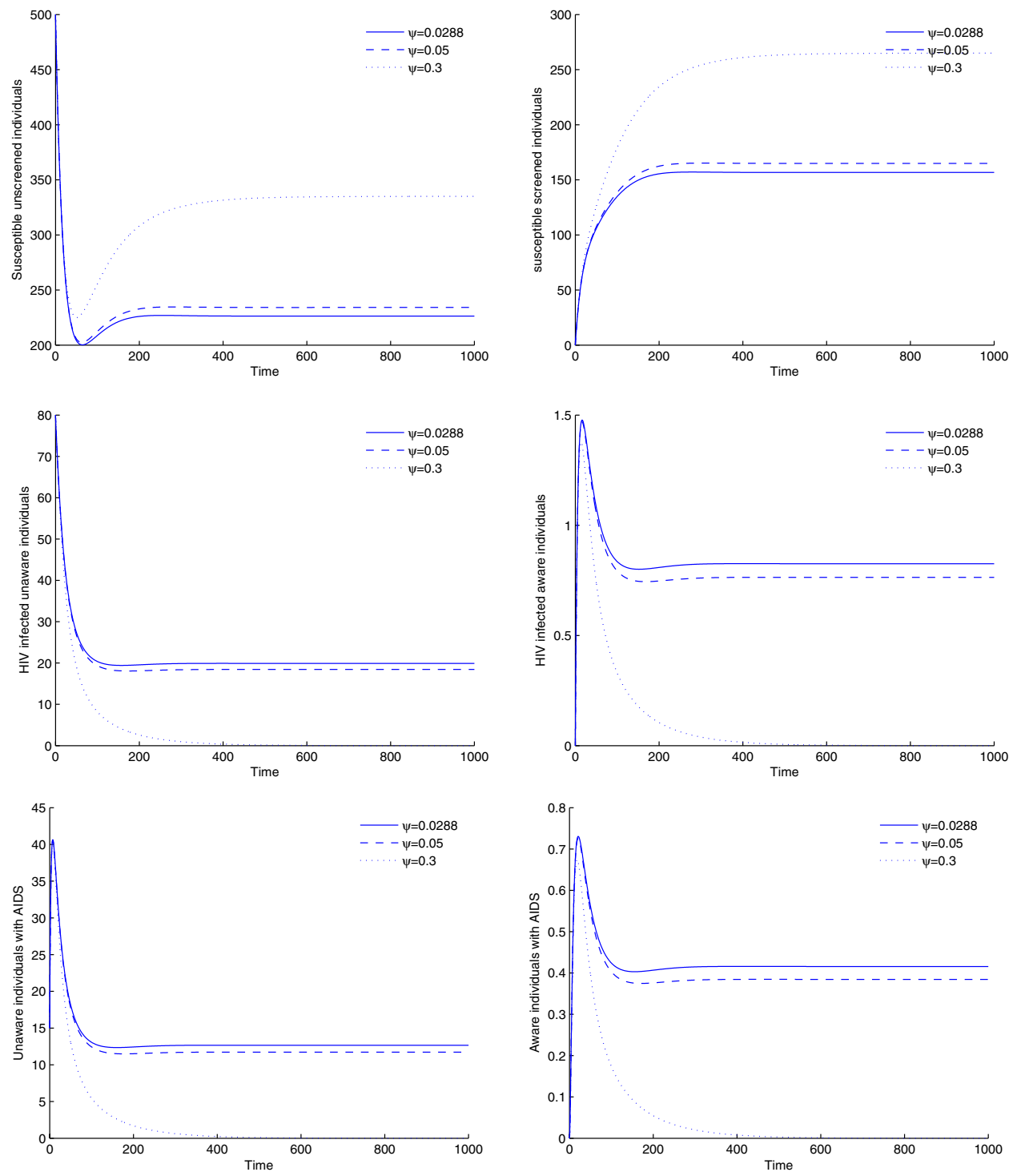

Fig. 12. Dynamics of the relevant variables of the system (2.1) for different values of $\psi$, the parameter that measures the efficacy of screening in reducing HIV transmission. Parameter values are given in Table 3 and initial conditions are in the text. Remaining variables go asymptotically to zero. For more information, see text.

\section{Conclusions}

We proposed a coinfection model for HCV and HIV infections, that includes treatment for both diseases, unawareness and awareness if HIV infection, and the use of condom. We studied the local stability of the disease-free equilibria for the full 
model and for the two submodels (HCV only and HIV only submodels). Bifurcation diagrams were sketched for relevant parameters, such as the probabilities that a contact will result in a HIV or an HCV infection. Numerical examples are used to illustrate the change of the dynamical behavior of certain relevant parameters. The results obtained suggest that specific measures should be considered in order to reduce HIV infection, such as: distributing more condoms to individuals and try to forward the message that condoms should be used during anal intercourse (though this might not be well accepted in MSM population due to unprotected sex negotiation); develop campaign in order to warn individuals about the consequences of having many sexual partners; continuing treatment for AIDS and pursuing the investigation of new and better drugs to combat the virus and regular screening. Considering HCV infection, treatment is also highly recommended as well as other measures (e.g., more informational campaign about the disease) in order to decrease the number of infectious and of chronic carriers. MSM population is at risk of HCV reinfection following successful treatment and documented clearance of HCV, they should be warned about this important risk. Future work will consider the effect of needle sharing in the case of HIV and HCV transmission rates, and an application of the model to real Portuguese data, with corresponding estimation of parameter values.

\section{Acknowledgments}

Authors wish to thank Fundação Gulbenkian, through Prémio Gulbenkian de Apoio à Investigação 2003, and the European Regional Development Fund through the program COMPETE and the Portuguese Government through the FCT - Fundação para a Ciência e a Tecnologia, under the project PEst-C/MAT/UI0144/2013, for financial support. The research of A.C. was supported by a FCT grant with reference SFRH/BD/96816/2013.

\section{References}

1. Wolff FH, Fuchs SC, Barcellos NT, Falavigna M, Cohene M, Brand o ABM, Fuchs $\mathrm{FD}$, Risk factors for hepatitis $\mathrm{C}$ virus infection in individuals infected with the HIV, Digestive Liver Dis 40:460-467, 2008.

2. GAT, Guia sobre hepatite C para as pessoas que vivem com o VIH: Testes, co -infecção e tratamento, Publicação GAT, 2009.

3. Rafikov M, Balthazar JM, von Bremen HF, Management of complex systems: Modeling the biological pest control, Biophys Rev Lett 3(1-2):241-256, 2008.

4. Rafikov M, Balthazar JM, On control and synchronization in chaotic and hyperchaotic systems via linear feedback control, Commun Nonlinear Sci Numer Simul 13:12461255, 2008.

5. Hyman JM, Li J, Stanley EA, Modelling the impact of screening and contact tracing in reducing the spread of HIV, Math Biosci 181:17-54, 2003.

6. Nyabadza F, A mathematical model for combating HIV/AIDS in Southern Africa: Will multiple strategies work? J Biol Syst 14:357-372, 2006. 
7. Garnett GP, An introduction to mathematical models of sexually transmitted disease epidemiology, Sex Transm Infect 78:7-12, 2002.

8. Tripathi A, Naresh R, Sharma D, Modelling the effect of screening of unaware infectives on the spread of HIV infection, Appl Math Comput 184:1053-1068, 2007.

9. Musekwa-Hove SD, Nyabadza F, The dynamics of an HIV/AIDS model with screened disease carriers, Comput Math Methods Med 10:287-305, 2009.

10. Thein HH, Yi Q, Dore GJ, Krahn MD, Natural history of hepatitis C virus infection in HIV infected individuals and the impact of HIV in the era of highly active antiretroviral therapy: A meta-analysis, AIDS 22:1979-1991, 2008.

11. Franciscus A, A Guide to: HIV/HCV Coinfection, Hepatitis C Support Project, 2012.

12. Van de Laar TJ, Van der Bij AK, Prins M, Bruisten SM, Brinkman K, Ruys TA, et al., Increase in HCV incidence among men who have sex with men in Amsterdam most likely caused by sexual transmission, J Infect Dis 196:230-238, 2007.

13. Fierer DS, Uriel AJ, Carriero DC, Klepper A, Dieterich DT, Mullen MP et al., Liver fibrosis during an outbreak of acute hepatitis $\mathrm{C}$ virus infection in HIV-infected men: A prospective cohort study, J Infect Dis 198:683-686, 2008.

14. Matthews GV, Hellard M, Kaldor J, Lloyd A, Dore GJ, Further evidence of HCV sexual transmission among HIV-positive men who have sex with men: Response to Danta et al., Aids 21:2112-2113, 2007.

15. Sulkowski MS, Viral hepatitis and HIV coinfection, J Hepat 48:353-367, 2008.

16. Danta M, Brown D, Bhagani S, Pybus OG, Sabin CA, Nelson M et al., Recent epidemic of acute hepatitis $\mathrm{C}$ virus in HIV positive men who have sex with men linked to high-risk sexual behaviours, Aids 21:983-991, 2007.

17. Van de Laar TJ, Matthews GV, Prinsa M, Dantad M, Acute Hepatitis C in HIVinfected men who have sex with men: An emerging sexually transmitted infection, Aids 24(12):1799-1812, 2010.

18. Capa L, Soriano V, Garcia-Samaniego J, Nunez M, Romero M, Cascarejo A et al., The magnitude of HCV genotype and coinfection with human immunodeficiency virus on CD4(+) and CD8(+) T-cell responses to hepatitis C virus, J Med Virol 79:503-510, 2007.

19. Danta M, Semmo N, Fabris P, Brown D, Pybus OG, Sabin CA et al., Impact of $\mathrm{HIV}$ on host-virus interactions during early hepatitis $\mathrm{C}$ virus infection, J Infect Dis 197:1558-1566, 2008.

20. Bhunu CP, Mushayabasa S, Modelling the transmission dynamics of HIV/AIDS and hepatitis C virus co-infection, HIV AIDS Rev 12:37-42, 2013.

21. Rockstroh JK, Influence of viral hepatitis on HIV infection, J Hepat 44:S25-S27, 2006.

22. Van den Driessche P, Watmough P, Reproduction numbers and sub-threshold endemic equilibria for compartmental models of disease transmission, Math Biosci 180:29-48, 2002.

23. Ermentrout B, XPPAUT® - The differential equations tool, version 5.98, 2006, Available at http://www.math.pitt.edu/ bard/xpp/xpp.html.

24. Chitnis N, Hyman JM, Cushing JM, Determining important parameters in the spread of malaria through the sensitivity analysis of a mathematical model, Bull Math Biol 70:1272-1296, 2008.

25. Abu-Raddad LJ, Schiffer JT, Ashley R, Mumtaz G, Alsallaq RA, Akala FA, Semini I, Riedner G, Wilson D, HSV-2 serology can be predictive of HIV epidemic potential and hidden sexual risk behavior in the Middle East and North Africa, Epidemics 2:173-182, 2010. 
26. Hollingsworth TD, Anderson RM, Fraser C, HIV-1 transmission, by stage of infection, $J$ Infect Dis 198:687-693, 2008.

27. Nyabadza F, Mukandavire Z, Modeling HIV/AIDS in the presence of an HIV testing and screening campaign, J Theor Biol 280:167-179, 2011.

28. Centers for Disease Control and Prevention, Division of Viral Hepatitis, Available at http://www.cdc.gov/hepatitis/hcv/pdfs/hepcgeneralfactsheet.pdf.

29. Weinhardt LS, Carey MP, Johnson BT, Bickham NL, Effects of HIV counseling and testing on sexual risk behavior: A meta-analytic review of published research, 19851997, Am J Public Health 89:1397-1405, 1999. 


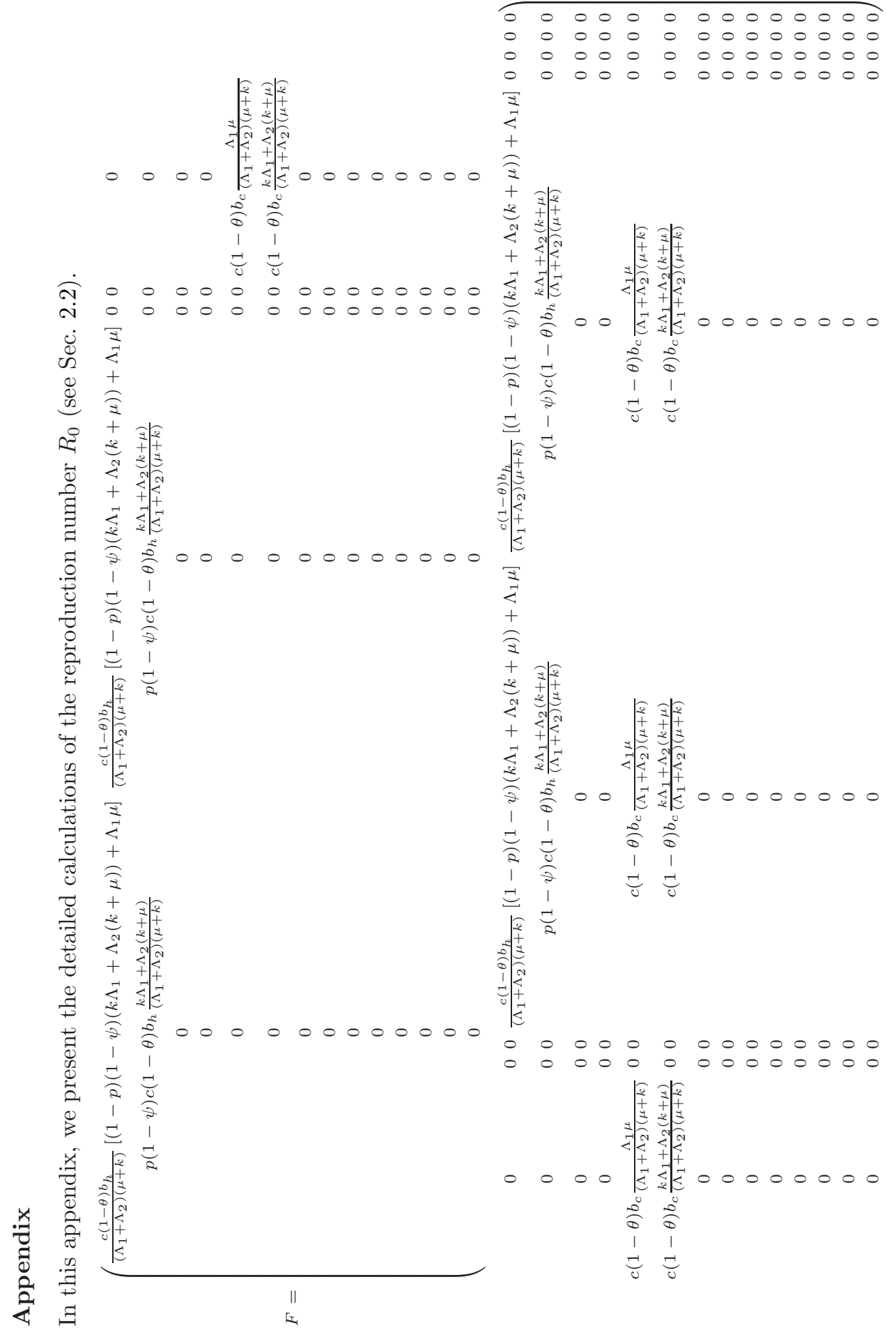




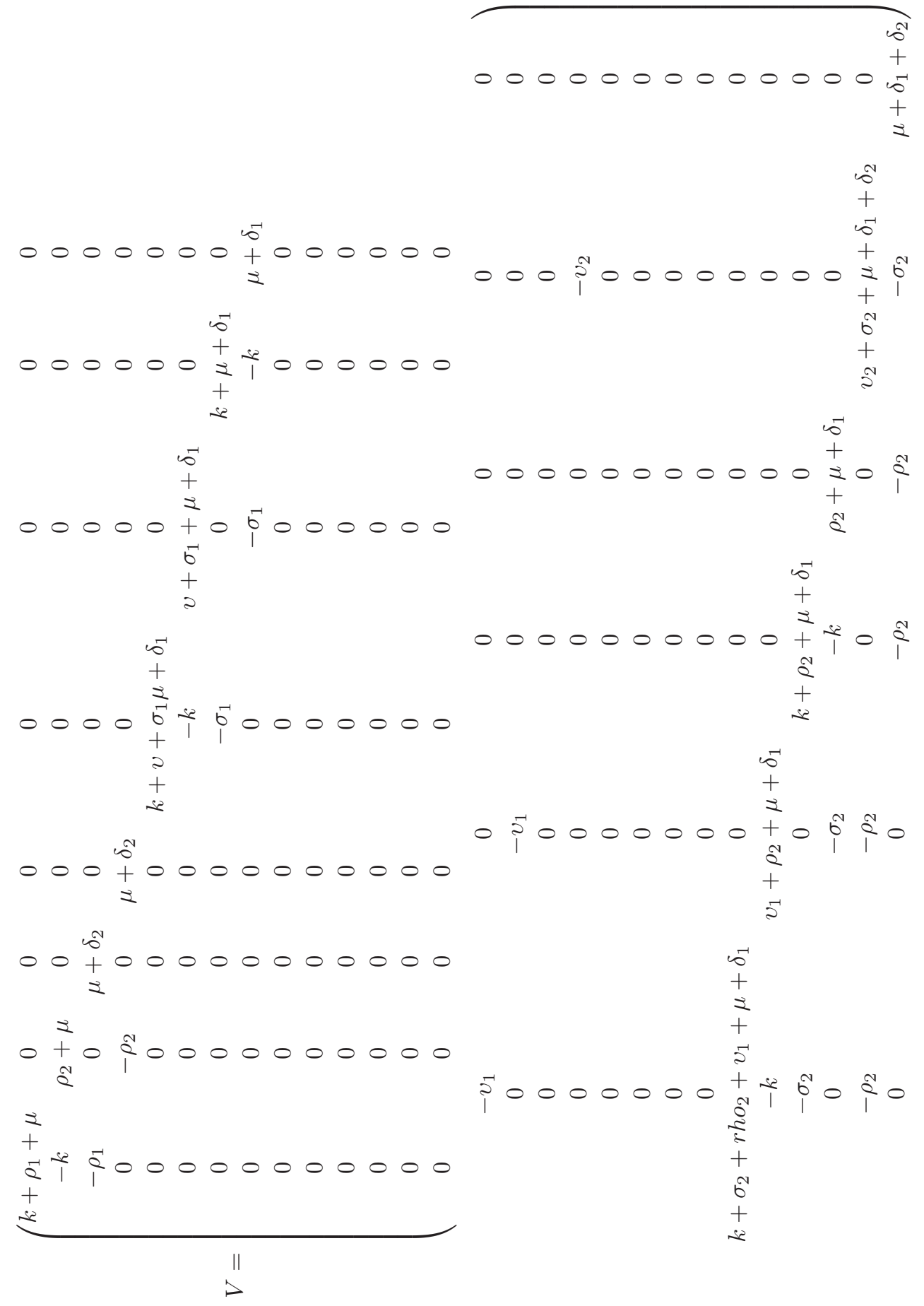




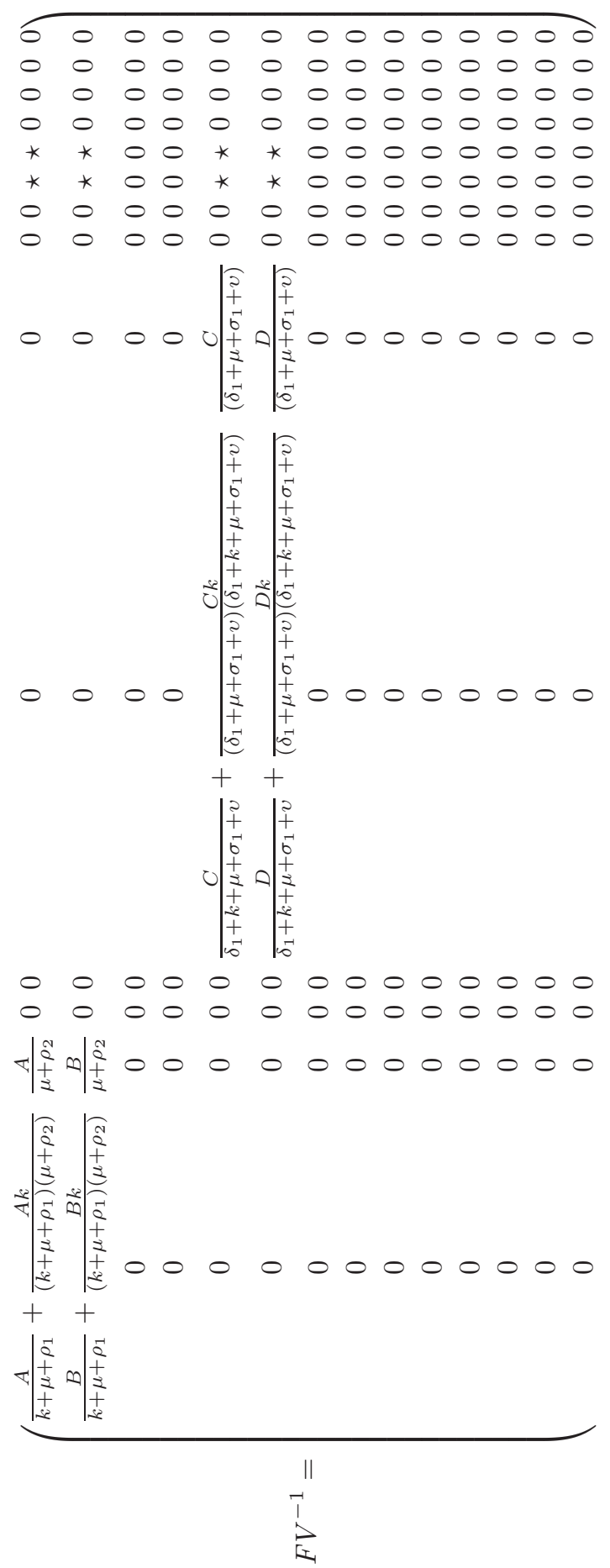


where $A=\frac{c(1-\theta) b_{h}}{\left(\Lambda_{1}+\Lambda_{2}\right)(\mu+k)}\left[(1-p)(1-\psi)\left(k \Lambda_{1}+\Lambda_{2}(k+\mu)\right)+\Lambda_{1} \mu\right], B=p(1-\psi) c(1-$ $\theta) b_{h} \frac{k \Lambda_{1}+\Lambda_{2}(k+\mu)}{\left(\Lambda_{1}+\Lambda_{2}\right)(\mu+k)}, C=c(1-\theta) b_{c} \frac{\Lambda_{1} \mu}{\left(\Lambda_{1}+\Lambda_{2}\right)(\mu+k)}$, and $D=c(1-\theta) b_{c} \frac{k \Lambda_{1}+\Lambda_{2}(k+\mu)}{\left(\Lambda_{1}+\Lambda_{2}\right)(\mu+k)}$. The formulas represented by $\star$ are irrelevant to compute the eigenvalues, so, for the sake of simplicity, we decided not to include those here.

In order to compute $R_{0}$, the basic reproduction number for the whole model (2.1), we need to compute the eigenvalues of the matrix $F V^{-1}$, since $R_{0}$ is the spectral radius of $F V^{-1}$, i.e., it is the dominant eigenvalue of this matrix.

Let us now proceed with the computation of the eigenvalues by calculating the determinant below:

$$
\begin{aligned}
F V^{-1}= & \left(\begin{array}{ccc}
\frac{A}{k+\mu+\rho_{1}}+\frac{A k}{\left(k+\mu+\rho_{1}\right)\left(\mu+\rho_{2}\right)}-\lambda & \frac{A}{\mu+\rho_{2}} \\
\frac{B}{k+\mu+\rho_{1}}+\frac{B k}{\left(k+\mu+\rho_{1}\right)\left(\mu+\rho_{2}\right)} & \frac{B}{\mu+\rho_{2}}-\lambda \\
0 & 0 & \\
0 & 0 & 0 \\
0 & & 0 \\
0 & & \\
\frac{C}{\delta_{1}+k+\mu+\sigma_{1}+v}+\frac{C k}{\left(\delta_{1}+\mu+\sigma_{1}+v\right)\left(\delta_{1}+k+\mu+\sigma_{1}+v\right)}-\lambda & \frac{C}{\left(\delta_{1}+\mu+\sigma_{1}+v\right)} \\
\frac{D}{\delta_{1}+k+\mu+\sigma_{1}+v}+\frac{D k}{\left(\delta_{1}+\mu+\sigma_{1}+v\right)\left(\delta_{1}+k+\mu+\sigma_{1}+v\right)} & \frac{D}{\left(\delta_{1}+\mu+\sigma_{1}+v\right)}-\lambda
\end{array}\right) .
\end{aligned}
$$

As $F V^{-1}$ is a matrix of the form: $\left[\begin{array}{cc}U_{1} & 0 \\ 0 & U_{2}\end{array}\right]$, then its eigenvalues are the ones of matrices $U$ and $V$, where:

$$
\begin{aligned}
U_{1} & =\left(\begin{array}{cc}
\frac{A}{k+\mu+\rho_{1}}+\frac{A k}{\left(k+\mu+\rho_{1}\right)\left(\mu+\rho_{2}\right)}-\lambda & \frac{A}{\mu+\rho_{2}} \\
\frac{B}{k+\mu+\rho_{1}}+\frac{B k}{\left(k+\mu+\rho_{1}\right)\left(\mu+\rho_{2}\right)} & \frac{B}{\mu+\rho_{2}}-\lambda
\end{array}\right) ; \\
U_{2} & =\left(\begin{array}{cc}
\frac{C}{\delta_{1}+k+\mu+\sigma_{1}+v}+\frac{C k}{\left(\delta_{1}+\mu+\sigma_{1}+v\right)\left(\delta_{1}+k+\mu+\sigma_{1}+v\right)}-\lambda & \frac{C}{\left(\delta_{1}+\mu+\sigma_{1}+v\right)} \\
\frac{D}{\delta_{1}+k+\mu+\sigma_{1}+v}+\frac{D}{\left(\delta_{1}+\mu+\sigma_{1}+v\right)\left(\delta_{1}+k+\mu+\sigma_{1}+v\right)} & \frac{D}{\left(\delta_{1}+\mu+\sigma_{1}+v\right)}-\lambda
\end{array}\right) .
\end{aligned}
$$

